\title{
Large trispectrum in two-field slow-roll inflation
}

\author{
Joseph Elliston, ${ }^{a}$ Laila Alabidi, ${ }^{b}$ Ian Huston, ${ }^{a}$ David Mulryne ${ }^{a}$ and \\ Reza Tavakol ${ }^{a}$ \\ ${ }^{a}$ Astronomy Unit, Queen Mary University of London, Mile End Road, London, UK \\ ${ }^{b}$ Yukawa Institute for Theoretical Physics, Kyoto University, Kyoto 606-8502, Japan \\ E-mail: j.elliston@qmul.ac.uk, laila@yukawa.kyoto-u.ac.jp, i.huston@qmul.ac.uk, \\ d.mulryne@qmul.ac.uk, r.tavakol@qmul.ac.uk
}

\begin{abstract}
We calculate the conditions required to produce a large local trispectrum during two-field slow-roll inflation. This is done by extending and simplifying the 'heat-map' approach developed by Byrnes et al. The conditions required to generate a large trispectrum are broadly the same as those that can produce a large bispectrum. We derive a simple relation between $\tau_{\mathrm{NL}}$ and $f_{\mathrm{NL}}$ for models with separable potentials, and furthermore show that $g_{\mathrm{NL}}$ and $\tau_{\mathrm{NL}}$ can be related in specific circumstances. Additionally, we interpret the heatmaps dynamically, showing how they can be used as qualitative tools to understand the evolution of non-Gaussianity during inflation. We also show how $f_{\mathrm{NL}}, \tau_{\mathrm{NL}}$ and $g_{\mathrm{NL}}$ are sourced by generic shapes in the inflationary potential, namely ridges, valleys and inflection points.
\end{abstract}

Keywords: Inflation, Non-Gaussianity, bispectrum, trispectrum 


\section{Introduction}

Observational constraints on the statistics of the primordial curvature perturbation provide a powerful test of inflationary models. For models driven by canonical scalar fields, the distribution of the curvature perturbation is extremely close to Gaussian at horizon crossing [1-4]. Moreover, after horizon crossing, the curvature perturbation is conserved for singlefield models [5-11]. In multi-field models, however, if isocurvature modes play a role, it can evolve, and this evolution must be followed (for example see [12-22]). As a result the statistics may become less Gaussian, and even sufficiently non-Gaussian to be detectable by future probes, such as the Planck satellite [23]. In this paper, we consider models in which the non-Gaussianity of the curvature perturbation can evolve to become large during a slow-roll inflationary phase [24-27]. Moreover, we focus on two-field separable potentials, for which analytic formulae are available for the non-Gaussianity parameters [25, 28-31].

The best constraints on local non-Gaussianity to date are provided by the analysis of data from the WMAP satellite. The bispectrum is parametrised by $f_{\mathrm{NL}}$, which is constrained as $-5<f_{\mathrm{NL}}<59$ at $95 \%$ C.L. [32]. $\tau_{\mathrm{NL}}$ and $g_{\mathrm{NL}}$ parametrise the trispectrum and are constrained as $-0.6<\tau_{\mathrm{NL}} / 10^{4}<3.3$ and $-7.4<g_{\mathrm{NL}} / 10^{5}<8.2$ [33, 34], with Ref. [35] finding the slightly different constraint $-5.4<g_{\mathrm{NL}} / 10^{5}<8.6$. Planck will improve on these constraints considerably, and in the absence of a detection is expected to give the bounds $\left|f_{\mathrm{NL}}\right|<5, \tau_{\mathrm{NL}}<560[36]$ and $\left|g_{\mathrm{NL}}\right|<1.6 \times 10^{5}$ [33]. If a given inflationary model predicts a magnitude of the $f_{\mathrm{NL}}, \tau_{\mathrm{NL}}$ or $g_{\mathrm{NL}}$ parameters greater than these forecast bounds, then we refer to such a prediction as 'observationally relevant'. This is in contrast to a non-Gaussianity that could in principle be measured by an ideal observation, which conservatively requires these parameters to have magnitudes greater than unity. Following the standard diction in the literature, we describe such models as producing a 'large' non-Gaussianity.

Our aims in this paper are as follows: First, to show the constraints on the initial and final conditions of the inflationary evolution that lead to large values of the $g_{\mathrm{NL}}$ and $\tau_{\mathrm{NL}}$ parameters, and to compare these to the constraints that are required to produce a large value of $f_{\mathrm{NL}}$. Secondly, to derive a simple relation between $\tau_{\mathrm{NL}}$ and $f_{\mathrm{NL}}$ for twofield slow-roll models with separable potentials, which is compatible with the well-known consistency relation [37]. We will also investigate under which conditions it is possible to deduce robust relations between $g_{\mathrm{NL}}$ and $\tau_{\mathrm{NL}}$. Thirdly, following earlier studies of the $f_{\mathrm{NL}}$ parameter $[38,39]$, we discuss the behaviour of $\tau_{\mathrm{NL}}$ and $g_{\mathrm{NL}}$ associated with generic features in inflationary potentials, namely ridges, valleys and inflection points. Finally, we demonstrate that, in addition to showing the spectrum of possible behaviours, our formalism can be used as a qualitative tool to give insight into how the non-Gaussianity parameters evolve during inflation.

Our method is an extension of the graphical approach employed by Byrnes et al. [27] to study the $f_{\mathrm{NL}}$ parameter. The method begins by re-casting the expressions for $f_{\mathrm{NL}}, \tau_{\mathrm{NL}}$ and $g_{\mathrm{NL}}$ as a sum of terms. Each term is the product of a combination of slow-roll parameters and a function of two other parameters related to the initial and final conditions satisfied by a given evolution. Since the combination of slow-roll parameters is guaranteed to be much less than unity, if the non-Gaussianity parameters are to be large, the functions must take values much greater than unity. One can therefore make 2D contour plots (or 'heatmaps') of these functions, and so identify the regions in parameter space which can give rise to large local non-Gaussianities. In this study, we have found that it is possible to simplify the expressions 
to such an extent that only one heat-map is required to understand the conditions for a large bi-spectrum, one additional heat-map is required for $\tau_{\mathrm{NL}}$, and three further heatmaps are required for $g_{\mathrm{NL}}$.

Recently, Peterson \& Tegmark have also undertaken a study of the bi- and tri-spectrum parameters [40-42] in the setting of slow-roll inflation, arriving at compact relationships between $\tau_{\mathrm{NL}}, g_{\mathrm{NL}}, f_{\mathrm{NL}}$ and the tilts of the curvature and isocurvature power spectra. Our approach is complimentary to their study, and provides new insights. For example, working directly with analytic expressions for $f_{\mathrm{NL}}, \tau_{\mathrm{NL}}$ and $g_{\mathrm{NL}}$ allows us to present more explicit formulae, and in particular helps us to understand the evolution of non-Gaussianity directly in terms of the shape of the inflationary potential. Our general conclusions are, however, very similar.

The paper is structured as follows: $\S 2$ provides some background material and reviews how analytic expressions for observable parameters can be derived using the $\delta N$ formalism. The expressions themselves are given in appendix A. $\S 3$ shows how we can simplify these expressions for the bi- and tri-spectra which we then analyse in $\S 4.1$ and $\S 4.2$ respectively. This analysis motivates three generic shapes in the inflationary potential which we study in $\S 5$, finding the peak values of $f_{\mathrm{NL}}, \tau_{\mathrm{NL}}$ and $g_{\mathrm{NL}}$ in each case. In $\S 6$ we give concrete examples which illustrate the findings of this paper. We conclude in $\S 7$.

\section{Background Theory}

We consider inflation driven by two canonical scalar fields $\phi_{i}$ with $i=1,2$, self-interacting through a potential $W\left(\phi_{1}, \phi_{2}\right)$. The scalar field equations of motion are

$$
\ddot{\phi}_{i}+3 H \dot{\phi}_{i}+W_{, i}=0,
$$

where a comma denotes partial differentiation with respect to the field $\phi_{i}$, dots are derivatives with respect to proper time $t$, and $H$ is the Hubble rate given by the Friedman equation

$$
3 M_{\mathrm{pl}}^{2} H^{2}=W+\frac{1}{2} \sum_{i} \dot{\phi}_{i}^{2} .
$$

We choose definitions of the slow-roll parameters as

$$
\begin{aligned}
\epsilon_{i} & =\frac{M_{\mathrm{pl}}^{2}}{2} \frac{W_{, i}^{2}}{W^{2}}, & \epsilon & =\sum_{i=1}^{2} \epsilon_{i}, \\
\eta_{i j} & =M_{\mathrm{pl}}^{2} \frac{W_{, i j}}{W}, & \xi_{i j k}^{2} & =M_{\mathrm{pl}}^{3} \sqrt{2 \epsilon} \frac{W_{, i j k}}{W} .
\end{aligned}
$$

Expressions for $\xi_{i j k}^{2}$ and $\eta_{i j}$ in the frame adapted to the field evolution, which we refer to as the kinematic frame, are given in appendix A. Inflation occurs when $\epsilon \lesssim 1$, and the so called 'slow-roll limit' is given by $\epsilon \ll 1$. In this limit the fields' kinetic energy can be neglected, and the field equations are well approximated by the slow-roll equations of motion

$$
3 H \dot{\phi}_{i}+W_{, i}=0, \quad 3 M_{\mathrm{pl}}^{2} H^{2}=W .
$$




\subsection{The Curvature Perturbation}

The primordial curvature perturbation on uniform density spatial hypersurfaces (see for example [43]) is denoted by $\zeta$.

The statistical properties of $\zeta$, which are constrained by observations, are commonly measured in terms of its power spectrum, bispectrum, and trispectrum, defined as

$$
\begin{aligned}
\left\langle\zeta_{\mathbf{k}_{1}} \zeta_{\mathbf{k}_{2}}\right\rangle & \equiv(2 \pi)^{3} \delta^{3}\left(\mathbf{k}_{1}+\mathbf{k}_{2}\right) \frac{2 \pi^{2}}{k_{1}{ }^{3}} \mathcal{P}_{\zeta}\left(k_{1}\right), \\
\left\langle\zeta_{\mathbf{k}_{1}} \zeta_{\mathbf{k}_{2}} \zeta_{\mathbf{k}_{3}}\right\rangle & \equiv(2 \pi)^{3} \delta^{3}\left(\mathbf{k}_{1}+\mathbf{k}_{2}+\mathbf{k}_{3}\right) \mathcal{B}_{\zeta}\left(k_{1}, k_{2}, k_{3}\right), \\
\left\langle\zeta_{\mathbf{k}_{1}} \zeta_{\mathbf{k}_{2}} \zeta_{\mathbf{k}_{3}} \zeta_{\mathbf{k}_{4}}\right\rangle & \left.\equiv(2 \pi)^{3} \delta^{3}\left(\mathbf{k}_{1}+\mathbf{k}_{2}\right)+\mathbf{k}_{3}+\mathbf{k}_{4}\right) \mathcal{T}_{\zeta}\left(k_{1}, k_{2}, k_{3}, k_{4}\right),
\end{aligned}
$$

respectively. For the local shape of non-Gaussianity, it is convenient to further parametrise the bispectrum and trispectrum in terms of the dimensionless parameters, $f_{\mathrm{NL}}, \tau_{\mathrm{NL}}$ and $g_{\mathrm{NL}}$, defined by

$$
\begin{aligned}
\mathcal{B}_{\zeta}\left(k_{1}, k_{2}, k_{3}\right)= & \frac{6}{5} f_{\mathrm{NL}}\left[P_{\zeta}\left(k_{1}\right) P_{\zeta}\left(k_{2}\right)+3 \text { perms }\right] \\
\mathcal{T}_{\zeta}\left(k_{1}, k_{2}, k_{3}, k_{4}\right)= & \tau_{\mathrm{NL}}\left[P_{\zeta}\left(k_{13}\right) P_{\zeta}\left(k_{3}\right) P\left(k_{4}\right)+11 \text { perms }\right] \\
& +\frac{54}{25} g_{\mathrm{NL}}\left[P_{\zeta}\left(k_{2}\right) P_{\zeta}\left(k_{2}\right) P_{\zeta}\left(k_{4}\right)+3 \text { perms }\right] .
\end{aligned}
$$

\subsection{The $\delta N$ formalism}

In order to follow the evolution of $\zeta$ on super-horizon scales, and calculate its statistics, we employ the separate universe approach to perturbation theory [6, 44], and the $\delta N$ formalism $[14,28,45]$. In this approach, spatial gradients are neglected on scales greater than the horizon size, and each spatial point is assumed to evolve as a separate FRW universe. Choosing a flat initial slicing at the time at which observational scales crossed the cosmological horizon, $t=t^{*}$, and a later uniform density (constant $H$ ) slicing at $t=t_{c}$, then $\zeta$ on the final slicing can be equated with the excess expansion, $\zeta=\delta N$.

During slow-roll inflation, field velocities are functions of field positions. Taking this to be a good approximation at horizon crossing, the subsequent number of efolds undergone by any 'separate universe' is a function purely of the initial field values on the flat slicing, $N\left(\phi_{1}^{*}, \phi_{2}^{*}\right)$, even if the evolution subsequently evolves away from slow-roll. One therefore finds that

$$
\zeta \equiv \delta N=N_{i} \delta \phi_{i}^{*}+\frac{1}{2 !} N_{i j} \delta \phi_{i}^{*} \delta \phi_{j}^{*}+\frac{1}{3 !} N_{i j k} \delta \phi_{i}^{*} \delta \phi_{j}^{*} \delta \phi_{k}^{*}+\ldots,
$$

where here and throughout we employ the summation convention, $N$ is the number of efolds from $t^{*}$ to $t_{c}$, a subscript $i$ on $N$ represents a derivative with respect to the light fields at horizon crossing $\phi_{i}^{*}$, and $\delta \phi_{i}^{*}$ are the field fluctuations on a flat hypersurface at horizon crossing.

Using the expression for $\zeta$ from Eq. (2.8), and recalling Eqs. (2.5)-(2.7), one can write observational parameters in terms of the derivatives of $N$ [14, 28-30],

$$
\begin{gathered}
P=N_{i} N_{i} P_{*}, \quad n_{s}-1=\frac{2}{H^{*}} \frac{\dot{\phi}_{i}^{*} N_{i j} N_{j}}{\left(N_{k} N_{k}\right)^{2}}-2 \epsilon^{*}, \\
\frac{6}{5} f_{\mathrm{NL}}=\frac{N_{i} N_{j} N_{i j}}{\left(N_{k} N_{k}\right)^{2}}, \quad \tau_{\mathrm{NL}}=\frac{N_{i j} N_{i k} N_{j} N_{k}}{\left(N_{k} N_{k}\right)^{3}}, \quad \frac{54}{25} g_{\mathrm{NL}}=\frac{N_{i j k} N_{i} N_{j} N_{k}}{\left(N_{k} N_{k}\right)^{3}}
\end{gathered}
$$


where $n_{s}$ is the spectral index, $P_{*}=H_{*}^{2} /(2 \pi)^{2}$, and where we have assumed the field fluctuations to be Gaussian at horizon crossing, which we recall is an excellent approximation for canonical fields $[2-4]$.

\subsection{Analytic formulae}

To calculate the observational parameters for a given inflation model we require the derivatives of $N$. These can always be calculated numerically, but analytic progress is possible only when the slow-roll equations of motion, Eq. (2.4), are a good approximation, and when a special 'separable' form of potential is assumed. This can either be a sum-separable potential of the form $W=\sum_{i} V_{i}\left(\phi_{i}\right)[15,25,29,46]$, or a product-separable form, $W=\Pi_{i} V_{i}\left(\phi_{i}\right)$ [47]. It is also possible to extend the analytic analysis slightly to models of the form $W=\left(\sum_{i} V_{i}\left(\phi_{i}\right)\right)^{1 / A}$ [48], where $A$ is an arbitrary constant. For simplicity, we will restrict our study to two-field sum- and product-separable potentials, labelling the fields $\phi_{1}=\phi$ and $\phi_{2}=\chi$. The two-field analytic formulae we employ for the derivatives of $N$, and for the observation parameters which are derived from them, are given in appendix A.

\subsection{The evolution of statistics and the adiabatic limit}

During inflation, if a given field space evolution follows a straight trajectory, then the statistics of the curvature perturbation will remain constant. Conversely, if the trajectory turns, the statistics will evolve $[18,24,25]$. During a turn, typically in the early stages, it is possible for the statistics of the curvature perturbation to become highly non-Gaussian [26, 27, 49]. If inflation were then to end suddenly, perhaps through a hybrid transition, it is possible that this large non-Gaussianity is preserved into the subsequent phases of the universe's evolution. Often, however, if the turn is completed, the non-Gaussianity returns to negligible levels. This possibility was emphasised in Ref. [31, 50].

This is not the only possibility, however. It is also possible for the bispectrum $[38,39$, $51,52]$ and trispectrum [53] to be large once a turn is completed, even after all isocurvature perturbations have decayed. If isocurvature modes decay, $\zeta$ and its statistics are subsequently conserved on super-horizon scales [7, 8], and this has recently been referred to as the adiabatic limit $[39,54]$. As discussed extensively in the literature (for example see $[13,15,17,39]$ ), reaching such a limit greatly simplifies the task of making observational predictions for an inflationary model. We emphasis, however, that there is no requirement that this occur, and if it does not, isocurvature modes will persist at the end of inflation [55]. The decay of isocurvature modes during slow-roll inflation occurs if the evolution reaches a regime which is effectively single field, such as a valley region with steep sides which force isocurvature perturbations to decay. One purpose of our study is to systematically identify all of the conditions which lead to a large $\tau_{\mathrm{NL}}$ and $g_{\mathrm{NL}}$ during slow-roll inflation, both in cases where isocurvature modes have, or have not, decayed.

If the evolution reaches a straight line trajectory along one of the axes, or the isocurvature modes decay, there is a considerable simplification of the formulae for $f_{\mathrm{NL}}, g_{\mathrm{NL}}$ and $\tau_{\mathrm{NL}}$ given in appendix $\mathrm{A}$. In particular, the terms involving $Z, \mathcal{A}$ and $\mathcal{B}^{2}$ (or $\mathcal{A}_{P}$ and $\mathcal{B}_{P}^{2}$ ) as defined in Eqs. (A.2), (A.6) and (A.12) (or eqs. (A.21) and (A.27)) tend to zero' ${ }^{1}$. These terms

\footnotetext{
${ }^{1}$ As discussed at length elsewhere [38], there is a possibility that, for sum-separable potentials, $Z$ does not go to zero. This exception only occurs if one of the fields, $\phi_{k}$, is completely orthogonal to the final straight line direction, and $V_{k}\left(\phi_{k}\right)$ tends to a constant as $V_{k}\left(\phi_{k}\right)^{\prime}$ tends to zero. Such a possibility can be avoided since we are free to reparametrise the potential and associate the problematic constant with another of the fields.
} 
vanishing is referred to as the Horizon Crossing Approximation (HCA) [56]. This greatly simplifies the expressions (A.14) and (A.28), allowing us to make much stronger statements about the relations between the observable non-Gaussianity parameters.

A final clarification is in order. Throughout this paper we calculate statistics on uniform density hypersurfaces. If, however, inflation ends suddenly, a further complication is that the surface on which inflation ends may not be a uniform density one. If this is not the case, an additional source of $\zeta$ will be produced [57], which will alter the statistics we calculate. We do not discuss this possibility further here, except briefly in $\S 6.1$. We note that in cases where all isocurvature modes decay there is no such possible additional effect.

\section{Analytic non-Gaussianity}

In this section we present the central results of our paper. We reformulate the analytic formulae for the non-Gaussianity parameters, which are reviewed in appendix A, into a set of terms, each of which is the product of a slow-roll suppressed part, and a function of two variables related to the initial and final conditions. Only the functions can be large, and so by plotting them as two-dimensional heatmaps, we can identify the initial and final conditions which lead to large non-Gaussianities. A key part of this procedure involves neglecting terms in these expressions that are always too small to contribute significantly to a large non-Gaussianity. We will see in subsequent sections, that these heatmaps are also useful for understanding the dynamical evolution of the non-Gaussianity parameters. Byrnes et al. [27] used a similar method to study the $f_{\mathrm{NL}}$ parameter. We first improve this bispectrum analysis by reducing to one the number of relevant heatmaps, and then consider the trispectrum.

\subsection{Variables}

We begin by defining the first important variable, the angle $\theta$ in the $\{\phi, \chi\}$ phase space in which the inflationary trajectory evolves. This can be done in terms of the slow-roll parameters $\epsilon_{\phi}$ and $\epsilon_{\chi}$, defined in Eq. (2.3), as

$$
\frac{\epsilon_{\phi}}{\epsilon}=\cos ^{2} \theta, \quad \frac{\epsilon_{\chi}}{\epsilon}=\sin ^{2} \theta .
$$

Since we assume that both fields are monotonically decreasing (which follows from our use of the slow-roll equations of motion), $\theta$ is constrained to lie in the range $0 \leq \theta \leq \pi / 2$.

The $\delta N$ expressions, given in appendix A, also involve the quantities $u$ and $v$, defined in Eqs. (A.1) and (A.17), which lie in the range zero to one. It proves convenient, therefore, to define a second angle, $\alpha$, in terms of these quantities as

$$
u=\cos ^{2} \alpha, \quad v=\sin ^{2} \alpha .
$$

We note that in the product-separable case, $\alpha=\theta$. The situation is not so simple in the sum-separable case, as we shall discuss in $\S 4.1$.

Substituting these definitions into the expressions for the non-Gaussianity parameters, Eqs. (A.16) and (A.30), we can eliminate the variables $u, v, \epsilon_{\phi}$ and $\epsilon_{\chi}$ in favour of $\alpha, \theta$ and $\epsilon$. The observables are then only functions of $\alpha, \theta$ and $\theta^{*}$, as well as $\epsilon$ and the other slow-roll parameters. Some of these slow-roll parameters are evaluated at horizon crossing, whilst others are evaluated on a later constant energy density hypersurface, usually labelled ' $c$ '. In the following we can drop this label without ambiguity, since within the $\delta N$ formalism all quantities evaluated after horizon crossing are calculated on a uniform density hypersurface. 
Quantities without a ' $*$ ' label are therefore assumed to be calculated on this later-time uniform density hypersurface.

\subsection{Bispectrum}

For the $f_{\mathrm{NL}}$ parameter the procedure outlined above leads to the expressions

$$
\begin{array}{ll}
\frac{6}{5} f_{\mathrm{NL}}=f_{1} \epsilon^{*}-f_{2} \eta_{s s}^{*}+f_{3} \eta_{\sigma s}^{*}+2 f \Omega\left(\eta_{s s}-\epsilon\right) & \\
\frac{6}{5} f_{\mathrm{NL}}=-f_{2} \eta_{s s}^{*}+f_{3} \eta_{\sigma s}^{*}+2 f \eta_{s s} & \text { - Sum separable } \\
\end{array}
$$

where, similarly to Ref. [27], we have defined the functions $f\left(\alpha, \theta^{*}\right), f_{i}\left(\alpha, \theta^{*}\right), \Lambda$ and $\Omega$ as

$$
\begin{aligned}
f & =\frac{\sin ^{2} 2 \alpha}{4 \Lambda^{2}}\left(\cos ^{2} \alpha-\cos ^{2} \theta^{*}\right)^{2}, & f_{2} & =\frac{1}{\Lambda^{2}}\left(\cos ^{6} \alpha \sin ^{4} \theta^{*}+\sin ^{6} \alpha \cos ^{4} \theta^{*}\right), \\
f_{1} & =\frac{\sin ^{2} 2 \theta^{*}}{2 \Lambda}, & f_{3} & =\frac{\sin 2 \theta^{*}}{2 \Lambda^{2}}\left(\cos ^{6} \alpha \sin ^{2} \theta^{*}-\sin ^{6} \alpha \cos ^{2} \theta^{*}\right), \\
\Lambda & =\cos ^{4} \alpha \sin ^{2} \theta^{*}+\sin ^{4} \alpha \cos ^{2} \theta^{*}, & \Omega & =\frac{W^{2}}{W_{*}^{2}} \frac{\sin ^{2} 2 \theta}{\sin ^{2} 2 \alpha} .
\end{aligned}
$$

Since $0 \leq \Omega \leq 1^{2}$, the functions $f_{1 \rightarrow 3}$ and $f$ all multiply quantities of $\mathcal{O}(\epsilon)$ or smaller, and so a necessary, though not sufficient, condition for $f_{\mathrm{NL}}$ to be large is that the magnitude of one or more of these functions is large. Our analysis, therefore, identifies only the conditions for which it is possible to produce a large $f_{\mathrm{NL}}$ during slow-roll inflation.

Because we are only interested in cases where $f_{\mathrm{NL}}$ can be large, the expressions (3.3) may be further simplified by noting that $\left|f_{1}\right|$ is bounded by order of unity and so the term $f_{1} \epsilon^{*}$ is negligible. Similarly, the term $f_{3} \eta_{\sigma s}^{*}$ is negligible and can be dropped. This latter result follows by recalling that we have the freedom to interchange between $\eta_{\sigma \sigma}, \eta_{\sigma s}$ and $\eta_{s s}$ via the relations

$$
\begin{array}{ll}
\eta_{\sigma s}=\frac{1}{2} \tan 2 \theta\left(\eta_{s s}-\eta_{\sigma \sigma}\right) & - \text { Sum separable } \\
\eta_{\sigma s}=\frac{1}{2} \tan 2 \theta\left(\eta_{s s}-\eta_{\sigma \sigma}+2 \epsilon\right) & \text { - Product separable. }
\end{array}
$$

Considering first the sum-separable case and expanding $f_{3} \eta_{\sigma s}^{*}$ as

$$
f_{3} \eta_{\sigma s}^{*}=\left[\sin 2 \theta^{*} f_{3}\right] \eta_{\sigma s}^{*}+\left[\frac{1}{2}\left(1-\sin 2 \theta^{*}\right) \tan 2 \theta^{*} f_{3}\right]\left(\eta_{s s}^{*}-\eta_{\sigma \sigma}^{*}\right),
$$

our result follows from the fact the the terms in square brackets can never become larger than order of unity. This procedure works because $\eta_{\sigma s}^{*}$ tends to zero in the limits of $\theta^{*} \rightarrow 0, \pi / 2$ which counters the divergence in $f_{3}$. Since Eq. (3.6) has the same form for both sum and product-separable potentials, we may neglect the term $f_{3} \eta_{\sigma s}^{*}$ in both cases.

${ }^{2}$ This follows by using (A.1) and the associated definitions found in appendix A to find

$$
\Omega=\frac{W^{2} \epsilon_{\phi} \epsilon_{\chi}}{\left(U^{*} \epsilon+V \epsilon_{\phi}-U \epsilon_{\chi}\right)\left(V^{*} \epsilon-V \epsilon_{\phi}+U \epsilon_{\chi}\right)} \leq \frac{W^{2} \epsilon_{\phi} \epsilon_{\chi}}{\left(U \epsilon+V \epsilon_{\phi}-U \epsilon_{\chi}\right)\left(V \epsilon-V \epsilon_{\phi}+U \epsilon_{\chi}\right)}=1,
$$

where the second inequality follows using $U \leq U^{*}$ and $V \leq V^{*}$. 
A final simplification follows by noting that $f_{2}=1+f$. We arrive at the extremely simple approximate expressions for $f_{\mathrm{NL}}$

$$
\begin{array}{ll}
\frac{6}{5} f_{\mathrm{NL}} \simeq f\left[-\eta_{s s}^{*}+2 \Omega\left(\eta_{s s}-\epsilon\right)\right] & \text { - Sum separable } \\
\frac{6}{5} f_{\mathrm{NL}} \simeq f\left[-\eta_{s s}^{*}+2 \eta_{s s}\right] & \text { - Product separable }
\end{array}
$$

where $f$ is positive definite. These simpler expressions make it transparent that the condition for $f_{\mathrm{NL}}$ to be large is that $f \gg 1$. We emphasise that these approximate expressions will be extremely accurate when $f_{\mathrm{NL}}>1$.

\subsection{Trispectrum}

A similar analysis can be performed for the $\tau_{\mathrm{NL}}$ and $g_{\mathrm{NL}}$ parameters. For sum-separable potentials we find

$$
\begin{gathered}
\tau_{\mathrm{NL}}=\tau_{1} \eta_{s s}^{* 2}-\tau_{2} \eta_{s s}^{*} \eta_{\sigma s}^{*}+\tau_{3} \eta_{\sigma s}^{* 2}-\tau_{4} \epsilon^{*} \eta_{s s}^{*}+\tau_{5} \epsilon^{*} \eta_{\sigma s}^{*}+\tau_{6} \epsilon^{* 2} \\
-\tau_{7} \Omega \eta_{s s}^{*}\left(\eta_{s s}-\epsilon\right)-\tau_{8} \Omega \eta_{\sigma s}^{*}\left(\eta_{s s}-\epsilon\right)+\tau_{9} \Omega \epsilon^{*}\left(\eta_{s s}-\epsilon\right)+4 \tau \Omega^{2}\left(\eta_{s s}-\epsilon\right)^{2} \\
\frac{54}{50} g_{\mathrm{NL}}=\tau_{1} \eta_{s s}^{* 2}-\tau_{2} \eta_{s s}^{*} \eta_{\sigma s}^{*}+\tau_{3} \eta_{\sigma s}^{* 2}-\frac{1}{4} \tau_{4} \epsilon^{*} \eta_{s s}^{*}+\frac{1}{4} \tau_{5} \epsilon^{*} \eta_{\sigma s}^{*}+\frac{1}{4} \tau_{2} \xi_{s s s}^{*} 2-\frac{1}{2} \tau_{3} \xi_{\sigma s s}^{*} 2 \\
-\frac{3}{4} \tau_{7} \Omega \eta_{s s}^{*}\left(\eta_{s s}-\epsilon\right)-\frac{3}{4} \tau_{8} \Omega \eta_{\sigma s}^{*}\left(\eta_{s s}-\epsilon\right) \\
+g_{1} \Omega^{3 / 2}\left(\xi_{s s s}^{2}-2 \eta_{\sigma s}\left(\eta_{s s}+\epsilon\right)\right)+4 g_{3} \Omega \frac{W}{W^{*}} \cos 2 \theta \eta_{s s}\left(\eta_{s s}-\epsilon\right)
\end{gathered}
$$

and for the product-separable potentials we find

$$
\begin{gathered}
\tau_{\mathrm{NL}}=\tau_{1} \eta_{s s}^{* 2}-\tau_{2} \eta_{s s}^{*} \eta_{\sigma s}^{*}+\tau_{3} \eta_{\sigma s}^{* 2}-\tau_{7} \eta_{s s}^{*} \eta_{s s}-\tau_{8} \eta_{\sigma s}^{*} \eta_{s s}+4 \tau \eta_{s s}^{2}, \\
\frac{54}{50} g_{\mathrm{NL}}=\tau_{1} \eta_{s s}^{* 2}-\tau_{2} \eta_{s s}^{*} \eta_{\sigma s}^{*}+\tau_{3} \eta_{\sigma s}^{* 2}+\tau_{3} \epsilon^{*} \eta_{s s}^{*}+\frac{1}{4} \tau_{2} \xi_{s s s}^{*}{ }^{2}-\frac{1}{2} \tau_{3} \xi_{\sigma s s}^{*}{ }^{2} \\
-\frac{3}{4} \tau_{7} \eta_{s s}^{*} \eta_{s s}-\frac{3}{4} \tau_{8} \eta_{\sigma s}^{*} \eta_{s s}+g_{1}\left(\xi_{s s s}^{2}-2 \eta_{\sigma s} \eta_{s s}\right)+4 g_{2} \eta_{s s}^{2}
\end{gathered}
$$

where the various functions occurring in these expressions are defined as

$$
\begin{aligned}
\tau_{1} & =\frac{1}{\Lambda^{3}}\left(\cos ^{8} \alpha \sin ^{6} \theta^{*}+\sin ^{8} \alpha \cos ^{6} \theta^{*}\right), & \tau_{4} & =2 f_{1} f_{2}, \\
\tau_{2} & =\frac{\sin 2 \theta^{*}}{\Lambda^{3}}\left(\cos ^{8} \alpha \sin ^{4} \theta^{*}-\sin ^{8} \alpha \cos ^{4} \theta^{*}\right), & \tau_{5} & =2 f_{1} f_{3}, \\
\tau_{3} & =\frac{f_{1}}{2 \Lambda^{2}}\left(\cos ^{8} \alpha \sin ^{2} \theta^{*}+\sin ^{8} \alpha \cos ^{2} \theta^{*}\right), & \tau_{6} & =f_{1}^{2}, \\
\tau_{7} & =\frac{4 f}{\Lambda}\left(\cos ^{2} \alpha \sin ^{2} \theta^{*}+\sin ^{2} \alpha \cos ^{2} \theta^{*}\right), & \tau_{9} & =4 f_{1} f, \\
\tau_{8} & =-\frac{\sin 2 \theta^{*} \sin ^{2} 2 \alpha}{2 \Lambda^{2}}\left(\cos ^{2} \alpha-\cos ^{2} \theta^{*}\right), & \tau & =\frac{f \sin ^{2} 2 \alpha}{4 \Lambda}, \\
g_{1} & =g_{3} \sin 2 \alpha, \quad g_{2}=g_{3} \cos 2 \alpha, & g_{3} & =-\frac{f}{2 \Lambda}\left(\cos ^{2} \alpha-\cos ^{2} \theta^{*}\right) .
\end{aligned}
$$

We can now proceed similarly as we did for the bispectrum and show that a number of these terms are negligible if the trispectrum parameters are large. Since the trispectrum functions pre-multiply quantities that are second order in slow-roll, in this case we neglect any functions that are never larger than 10, rather than order of unity. The details of this analysis can be found in appendix B. 


\subsection{1 $\tau_{\mathrm{NL}}$}

For $\tau_{\mathrm{NL}}$, we find the remarkably simple forms

$$
\begin{array}{ll}
\tau_{\mathrm{NL}} \simeq \mathcal{C}\left(\frac{6}{5} f_{\mathrm{NL}}\right)^{2}-\frac{12}{5} f_{\mathrm{NL}}\left(\eta_{s s}^{*}-f_{1} \epsilon^{*}\right) & \text { - Sum separable } \\
\tau_{\mathrm{NL}} \simeq \mathcal{C}\left(\frac{6}{5} f_{\mathrm{NL}}\right)^{2}-\frac{12}{5} f_{\mathrm{NL}} \eta_{s s}^{*} & \text { - Product separable }
\end{array}
$$

where

$$
\mathcal{C}=\frac{\tau}{f^{2}}=\frac{\Lambda}{\left(\cos ^{2} \alpha-\cos ^{2} \theta^{*}\right)^{2}} .
$$

A further simplification can be made by noting that $\mathcal{C} \geq 1$ and that $f_{1}$ is at most of order unity. The second terms will therefore be suppressed relative to the first by at least $\mathcal{O}\left(\epsilon^{*}\right)$. We thus find

$$
\tau_{\mathrm{NL}} \simeq \mathcal{C}\left(\frac{6}{5} f_{\mathrm{NL}}\right)^{2}
$$

which is valid for both sum and product-separable potentials.

In ref. [33], the ratio between $\tau_{\mathrm{NL}}$ and $\left(\frac{6}{5} f_{\mathrm{NL}}\right)^{2}$ was parameterised by $A_{\mathrm{NL}}$. Peterson et al. subsequently showed that $A_{\mathrm{NL}}=1 / r_{c}^{2}$ for two-field models under slow-roll [41], where $r_{c}$ determines the fraction of the curvature perturbation which is sourced by a horizon crossing isocurvature mode. Our result, Eq. (3.16), is complementary to this analysis, explicitly showing the form of $r_{c}$ in terms of the dynamics of inflation for separable potentials.

\subsection{2 $\quad g_{\mathrm{NL}}$}

Unsurprisingly, $g_{\mathrm{NL}}$ does not simplify so neatly. In the product-separable case we find

$$
\frac{54}{50} g_{\mathrm{NL}} \simeq \tau_{\mathrm{NL}}\left(\frac{\eta_{s s}^{*}-\eta_{s s}}{\eta_{s s}^{*}-2 \eta_{s s}}\right)-\frac{6}{5} f_{\mathrm{NL}}\left(2 \eta_{s s}^{*}+\eta_{s s}\right)-g_{4} \xi_{s s s}^{* 2}+g_{1}\left[\xi_{s s s}^{2}-2 \eta_{\sigma s} \eta_{s s}\right]
$$

where $g_{4}=\frac{1}{4}\left(\tau_{3} \sin 2 \theta^{*} \cos 2 \theta^{*}-\tau_{2}\right)$. For sum-separable potentials we obtain two additional terms, and the expression takes the form

$$
\begin{aligned}
\frac{54}{50} g_{\mathrm{NL}} \simeq & \tau_{\mathrm{NL}}\left(\frac{\eta_{s s}^{*}-\Omega\left(\eta_{s s}-\epsilon\right)}{\eta_{s s}^{*}-2 \Omega\left(\eta_{s s}-\epsilon\right)}\right)-\frac{6}{5} f_{\mathrm{NL}}\left(2 \eta_{s s}^{*}+\Omega\left(\eta_{s s}-\epsilon\right)\right)-g_{4} \xi_{s s s}^{* 2} \\
& +g_{1} \Omega^{3 / 2}\left[\xi_{s s s}^{2}-2 \eta_{\sigma s}\left(\eta_{s s}+\epsilon\right)\right]-\frac{1}{2} f_{1} f \epsilon^{*} \eta_{s s}^{*} \\
& +4 g_{3} \Omega\left(\eta_{s s}-\epsilon\right)\left(\frac{W}{W^{*}} \cos 2 \theta \eta_{s s}-\Omega \cos 2 \alpha\left(\eta_{s s}-\epsilon\right)\right) .
\end{aligned}
$$

These formulae for $g_{\mathrm{NL}}$ are once again complimentary to those derived by Peterson et al. [41]. One advantage of our results is that because of their explicit nature, we can easily consider which shapes in the inflationary potential will cause the different terms in Eqs. (3.17) and (3.18) to become large. Furthermore, the Horizon Crossing Approximation is easily implemented in our formulae by taking $\Omega \rightarrow 0$.

\section{Analysis}

We now turn to the interpretation of the formulae we have presented in the previous section. 


\subsection{Bispectrum}

The expressions (3.8) imply that a necessary condition for $\left|f_{\mathrm{NL}}\right|>1$ is that $f \gg 1$. We can see when this occurs by plotting $f\left(\alpha, \theta^{*}\right)$, as shown in Fig. 1 .

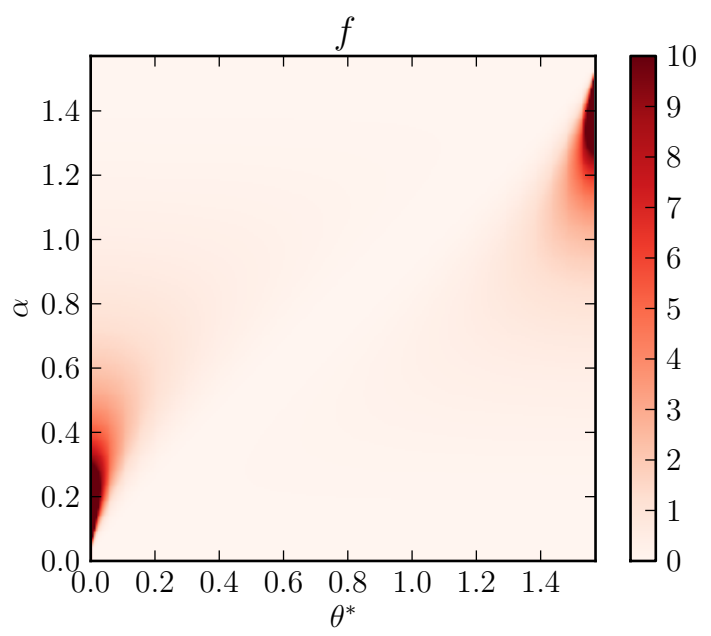

Figure 1. Heatmap of the function $f$. Since $f_{\mathrm{NL}}, \eta_{s s}$ and $\epsilon$ are symmetric under field exchange $\phi \leftrightarrow \chi$, the function $f$ must have the same symmetry. We can see this by inverting the heatmap though the point $\left(\theta^{*}, \alpha\right)=(\pi / 4, \pi / 4)$ which leaves $f$ invariant.

For product-separable potentials, $\alpha$ and $\theta$ are trivially related as $\alpha=\theta$. The initial (horizon crossing) value of $\alpha$ for product-separable potentials is therefore $\alpha_{\text {init }}=\theta^{*}$. For sum-separable potentials the same initial condition is found. This can be seen by taking the limit $c \rightarrow *$ in Eq. (A.1). This means that all separable inflationary models start on the diagonal of the heatmaps at horizon crossing. Since $f=0$ on the diagonal, we know that initially $f_{\mathrm{NL}}$ will be given by the various negligable terms that we have dropped in (3.8), and so $\left|f_{\mathrm{NL}}\right| \ll 1$ initially, as we expect [2].

As the model evolves from a given $\theta^{*}, \alpha$ varies such that the model traces a vertical line on the heatmap. For this trajectory to ever intercept one of the regions in which $f \gg 1$ the initial conditions must be such that the initial phase-space velocity is dominated by one of the two fields $\phi$ or $\chi$.

\subsubsection{The product-separable case}

As an example, let us take a product-separable model with $\theta^{*} \ll 1$, such that the horizon crossing conditions correspond to a position on the diagonal of the heatmap of $f$ in the lower left-hand corner. If the angle $\theta(=\alpha)$ increases towards $\pi / 2$, there can be a transient 'spike' in $f_{\mathrm{NL}}$ as the model passes through the region in which $f$ is large. If the trajectory turns back, so that $\theta$ decreases, it may well pass back through this region again and another transient signal in $f_{\mathrm{NL}}$ can be produced. Of course, whether or not a significant spike will occur is also dependent on the slow-roll parameters that define the model. The magnitude of this spike in $f_{\mathrm{NL}}$ increases as $\theta^{*}$ decreases towards zero. We must be careful with the interpretation, however, since our analysis presumes separable potentials and this places strong constraints on the possible modes of behaviour. If the initial field velocity is exactly aligned to one of 
the field axes then it will (classically) remain so indefinitely, since this implies $\theta=\theta^{*}$ always. Furthermore, for neighbouring initial conditions with sufficiently small values of $\theta^{*}$, one may find that $\theta$ will take longer than the required length of inflation (roughly 60 efolds) to grow sufficiently for there to be a significant enhancement of $f_{\mathrm{NL}}$. There will therefore be an upper bound on the value $f_{\mathrm{NL}}$ achievable by any such potential ${ }^{3}$.

\subsubsection{The sum-separable case}

To make progress with understanding the dynamics in the sum-separable case, it is necessary to understand the relationship between $\alpha$ and $\theta$. To do this we differentiate Eq. (3.2) to find

$$
\alpha^{\prime}=\frac{W}{W^{*}} \frac{\sin ^{2} 2 \theta}{\sin ^{2} 2 \alpha} \theta^{\prime},
$$

where a prime represents a derivative with respect to some time variable. The fractions in Eq. (4.1) are positive definite and so $\alpha$ increases as $\theta$ increases and vice versa.

Vacuum domination. More progress is possible by considering the vacuum-dominated limit, where $W \simeq W^{*}$. This is a good approximation for some models of inflation such as hybrid inflation. Eliminating the ratio $W / W^{*}$ from Eq. (4.1), we see that $\alpha \simeq \theta$. Moreover, one also finds $\Omega \simeq 1$ in this limit. Consequently, for vacuum-dominated sum-separable potentials, one may use the heatmaps in the same way as for product-separable potentials. Furthermore, we see that in this case the sum and product-separable formulae for $f_{\mathrm{NL}}$ are identical, apart from the presence of the slow-roll parameter $\epsilon$ in the sum-separable case. Thus for models with $\eta_{s s} \gg \epsilon$ there is a very near equivalence between product-separable potentials and vacuum-dominated sum-separable potentials in terms of their contribution to $f_{\mathrm{NL}}$.

General models. We now consider Eq. (4.1) for general sum-separable models without vacuum-domination. The ratio $W / W^{*}$ is initially unity and decreases towards zero. This ensures that, whilst the angle is monotonically varying, $\alpha$ lags behind $\theta$. Furthermore, the difference between $\alpha$ and $\theta$ will become more pronounced the smaller the ratio $W / W^{*}$. When this ratio goes to zero, we see that $\alpha$ remains constant despite any subsequent turning of the trajectory in phase space. Similarly, $\alpha$ will cease to evolve if either of the limits $\theta \rightarrow 0$ or $\theta \rightarrow \pi / 2$ are reached. Physically, these limits correspond to straight lines in the field phase space under which conditions it is well known that $\zeta$ does not evolve. We note that $\Omega$ is zero in any of these three limits and so we are only left with the $\eta_{s s}^{*}$ term in $f_{\mathrm{NL}}$. This is the approximate HCA formula for $f_{\mathrm{NL}}$. We note that since $\alpha \neq \theta$, it is quite possible for $f$ to be large when $\alpha$ becomes a constant, and so produce an observationally relevant constant $f_{\mathrm{NL}}$. For a given evolution, the final value of $\alpha$ is readily extracted once the initial and final field values are known, and hence one can check whether the correct value of $\alpha$ is reached in order to give a large non-Gaussianity.

The final case to consider is when the trajectory turns back on itself during its evolution. There is no barrier to constructing sum-separable potentials which exhibit this behaviour for particular evolutions. This will mean that the model moves up and then down a vertical line on the heatmap, perhaps many times. To fully quantitatively understand how this movement

\footnotetext{
${ }^{3}$ There will also be another upper bound on $\left|f_{\mathrm{NL}}\right|$ imposed by the quantum scatter of the field near horizon crossing which will prohibit the limit $\theta^{*} \rightarrow 0$ being physically realisable.
} 
occurs requires a knowledge of how the potential $W$ varies with $\theta$, which is necessarily modelspecific. However, it is possible to gain some more detailed intuition for how $\alpha$ varies with $\theta$ by rewriting Eq. (4.1) as

$$
h^{\prime}(\alpha)=\frac{W}{W^{*}} h^{\prime}(\theta),
$$

where we have defined the function $h(x)=4 x-\sin 4 x$, which is monotonic in $x$. One sees that $h(\alpha)$ and $h(\theta)$ increase and decrease simultaneously and hence the velocity $h^{\prime}(\alpha)$ is always smaller than the velocity $h^{\prime}(\theta)$. Since $W / W^{*}$ is constantly decreasing then so is the range of values of $h(\alpha)$ which the evolution can reach. Ultimately $h^{\prime}(\alpha) \rightarrow 0$ and $h(\alpha)$ takes a constant value. Since $h(\alpha)$ is monotonic in $\alpha$ we see that restricting the range of $h(\alpha)$ translates into introducing 'excluded regions' at the top and bottom of the heatmaps, the size of which will grow as the potential drops, and ultimately the whole of the heatmap will be excluded except for the final value of $\alpha$. Once again, for a given evolution the final value of $\alpha$ can be readily calculated, and one can check if it is in the observationally relevant regime.

\subsubsection{The role of $\Omega$}

The value of $\Omega$ determines which of the terms $\eta_{s s}^{*}$ or $\eta_{s s}$ dominates in Eq. (3.8). For productseparable or vacuum-dominated sum-separable potentials, $\Omega$ is effectively fixed to be unity, and so which term dominates depends on how $\eta_{s s}$ evolves during the evolution. On the other hand, if $\Omega \rightarrow 0$ then the $\eta_{s s}^{*}$ term will dominate. It is instructive, therefore, to think of inflationary models belonging to one of two classes, those for which $\Omega \simeq 1$ throughout and those for which $\Omega \rightarrow 0$ at some point. We now briefly consider each of these cases in turn.

Evolutions for which $\Omega \simeq 1$. These models either have product-separable or vacuumdominated sum-separable potentials. In the simplest cases of interest, such as falling from a potential ridge or rolling into a vacuum dominated valley [38], the absolute value of the potential does not change significantly during this phase of evolution. If $\eta_{s s} \simeq \eta_{s s}^{*}$ then one finds $\frac{6}{5} f_{\mathrm{NL}} \simeq f \eta_{s s}^{*}$. Since $f$ is positive definite, we see that the sign of $f_{\mathrm{NL}}$ is the same as the sign of the isocurvature mass. This is in agreement with previous calculations [38] where a ridge shape $\left(\eta_{s s}<0\right)$ was shown to lead to a negative $f_{\mathrm{NL}}$ and a valley shape $\left(\eta_{s s}>0\right)$ was shown to lead to a positive $f_{\mathrm{NL}}$. If inflation does not end abruptly, for example by a hybrid transition, but the fields continue their evolution, then $f_{\mathrm{NL}}$ will continue to evolve until $\theta \rightarrow 0$ or $\theta \rightarrow \pi / 2$. In either of these limits $f \rightarrow 0$ and so $f_{\mathrm{NL}}$ is much smaller than unity.

Evolutions for which $\Omega \rightarrow 0$. These models naturally reach a limit in which $\alpha$ becomes constant, and the HCA becomes a good approximation. This may or may not coincide with a scenario in which the isocurvature perturbations decay and an adiabatic limit is reached. If an adiabatic limit is arrived at, then we can be certain that there will be no further evolution of $\zeta$ and its statistics. When $\Omega \rightarrow 0$ we find $\frac{6}{5} f_{\mathrm{NL}} \simeq-f \eta_{s s}^{*}$. Due to the minus sign, if we begin in a region with a negative isocurvature mass, for example on a ridge, then such a model will eventually produce a positive $f_{\mathrm{NL}}$, whereas if we begin in region with a positive isocurvature mass, then $f_{\mathrm{NL}}$ will ultimately reach a negative limiting value.

In summary, the positive $f_{\mathrm{NL}}$ that is marginally preferred by the WMAP data [32] can be generated in two possible ways. Either, the evolution must begin with a small $\theta^{*}$ (or one close to $\pi / 2$ ) and an initially negative isocurvature mass, and then evolve until $\alpha$ naturally takes a small constant value suitable to make $f$ large. Or alternatively, the evolution must 
evolve from a small $\theta^{*}$ (or one close to $\pi / 2$ ) during which time the isocurvature mass is positive, and some mechanism much interrupt the dynamics whilst $f$ is large.

\subsection{Trispectrum}

We now turn to analyse the trispectrum. Our aims are twofold. First, to understand the types of models and the initial and final conditions for which the observational non-Gaussianity parameters $\tau_{\mathrm{NL}}$ and $g_{\mathrm{NL}}$ can be large. Secondly, to infer if it is possible to relate these nonGaussianity parameters to one another, perhaps for specific classes of models. Such relations are potentially important in order to observationally exclude classes of models.

Referring to Eqs. (3.14) we see that $\tau_{\mathrm{NL}}$ can only be large when $\tau$ or $f$ are large. We plot these functions side-by-side in Fig. 2. The fact that both functions peak in similar regions of the parameter space means that the classes of models that are capable of producing a large $\tau_{\mathrm{NL}}$ are the same as the classes of models that can produce a large $f_{\mathrm{NL}}$.
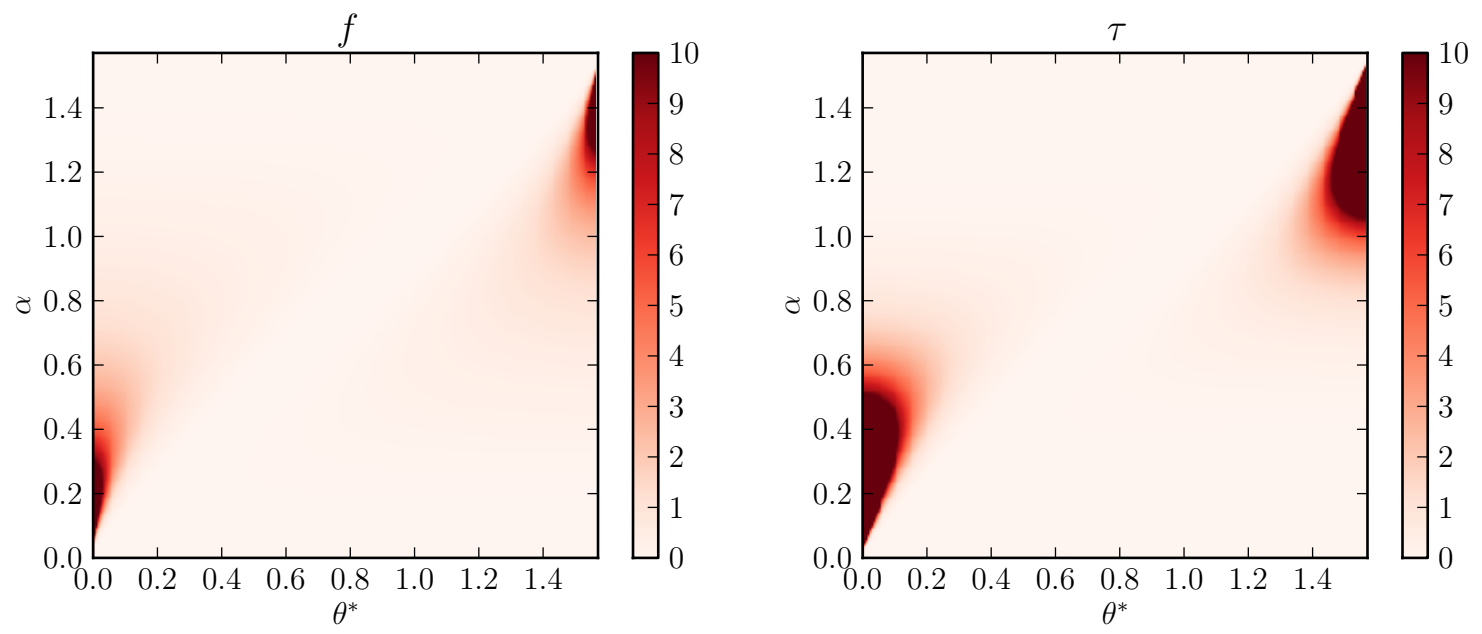

Figure 2. Heatmaps of $f$ and $\tau$ on the same scale. We note that both are large in the same regions of the parameter space.

\subsection{1 $\tau_{\mathrm{NL}}$ and $f_{\mathrm{NL}}$ relation}

For canonical scalar field inflation, $\tau_{\mathrm{NL}}$ and $f_{\mathrm{NL}}$ satisfy the condition [37]

$$
\tau_{\mathrm{NL}} \geq\left(\frac{6}{5} f_{\mathrm{NL}}\right)^{2}
$$

where equality occurs for single field inflation ${ }^{4}$. This is fully consistent with our result (3.16), once we recall that $\mathcal{C} \geq 1$. We plot $\mathcal{C}$ in Fig. 3. The interesting regions of this plot are those for which $\tau_{\mathrm{NL}}$ is not small and so $f^{2} \gg 1$ as shown in Fig. 2. We see that if a model has $\theta^{*} \ll 1$ and $\theta$ subsequently increases, then such a model will first enter the region in which $\mathcal{C} \gg 1$ and so $\tau_{\mathrm{NL}}$ will grow whilst $f_{\mathrm{NL}}$ remains small. It is unsurprising that $\tau_{\mathrm{NL}}$ evolves

\footnotetext{
${ }^{4}$ See also the recent work by Sugiyama [58] claiming that this equality is broken when calculations contributions are included from all loops.
} 
first, since being associated with a higher order moment, it will be more sensitive to outliers of the $\delta N$ distribution. For large $\alpha$, one can see that $\mathcal{C} \simeq 1$ to a very good degree of accuracy, and the single field relation becomes a good approximation. We note that $\tau_{\mathrm{NL}}$ deviates more from the single-field limit $\mathcal{C}=1$ when $\theta^{*}$ is fine-tuned to be closer to the $\theta^{*}=0$ axis.

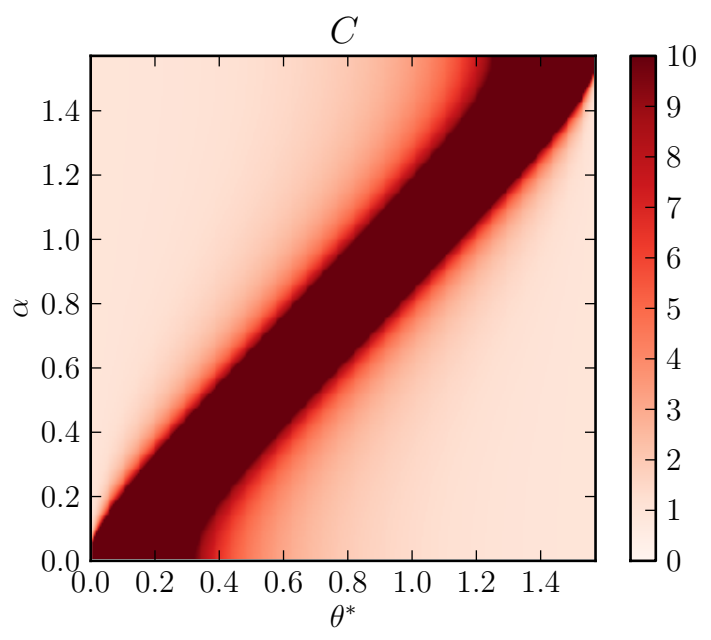

Figure 3. Heatmap of the function $\mathcal{C}$ illustrating the conditions required for a model to deviate from the single field consistency result for $\tau_{\mathrm{NL}}$. This is only physically interesting in the regions for which $f$ is large which are close to the sides of the heatmap. The region where $\mathcal{C} \gg 1$ overlaps with part- but not all of - the region where $f$ is large and one can see that this overlapping region is displaced from the sides of the heatmap.

\subsection{2 $\quad g_{\mathrm{NL}}$ and $\tau_{\mathrm{NL}}$ relations}

We now turn our attention to consider possible relations between $g_{\mathrm{NL}}$ and $\tau_{\mathrm{NL}}$.

The $\Omega=0$ case. First, we consider the case of non-vacuum dominated sum-separable potentials for evolutions which reach $\Omega=0$ such that all of the observational parameters have ceased to evolve. From Eq. (3.18) we see that

$$
\frac{54}{50} g_{\mathrm{NL}} \simeq \tau_{\mathrm{NL}}-\frac{3}{5} f_{\mathrm{NL}}\left(4 \eta_{s s}^{*}-f_{1} \epsilon^{*}\right)-g_{4} \xi_{s s s}^{*}{ }^{2} .
$$

The $f_{\mathrm{NL}}$ term in Eq. (4.4) is suppressed relative to the $\tau_{\mathrm{NL}}$ term by a relative factor of $f_{\mathrm{NL}}^{-1} \times \mathcal{O}\left(\epsilon^{*}\right)$ and so may be safely neglected. In cases were $\xi_{s s s}^{*}{ }^{2}$ can be neglected, for example in the absence of significant terms in the potential beyond quadratic order at horizon crossing, we find a relation between $\tau_{\mathrm{NL}}$ and $g_{\mathrm{NL}}$ as

$$
\frac{54}{50} g_{\mathrm{NL}} \simeq \tau_{\mathrm{NL}} .
$$

Next, let us look at the term $g_{4} \xi_{s s s}^{*}{ }^{2}$ and assess when it may be relevant. If an inflationary potential exists for which this term is important then it will have a signature such that $g_{\mathrm{NL}}$ deviates from Eq. (4.5). The heatmap for $g_{4}$ is plotted in Fig. 4 and we see that the areas in which $g_{4}$ is large are very small in comparison to the corresponding areas for the $\tau$ function. 
Practically speaking, this ensures that one has to tune the parameters of the model to a very high degree in order to access this region. In $\S 5$, we show that, in the interesting limit where $\theta^{*}$ and $\alpha$ are small, we can accurately approximate $2 g_{4} \simeq \theta^{*} \tau$. Thus a necessary (but not sufficient) condition for $\left|g_{\mathrm{NL}}\right|$ to be large is that $\theta^{*} \tau \gg 1$. In addition, for $g_{\mathrm{NL}}$ to deviate from Eq. (4.5) we require that $L=\theta^{*} \xi_{s s s}^{*} / 2 \eta_{s s}^{* 2}$ is not small. Potentials with $L \gg 1$ therefore have the capacity to generate $\left|g_{\mathrm{NL}}\right| \gg \tau_{\mathrm{NL}}$.

Considering a sum separable potential, in the case where $\theta^{*} \ll 1$, to leading order we find

$$
\eta_{s s}^{* 2}=\eta_{\chi}^{* 2}, \quad \xi_{s s s}^{* 2}=\xi_{\chi}^{* 2}, \quad \theta^{*}=\sqrt{\epsilon_{\chi}^{*} / \epsilon_{\phi}^{*}},
$$

where we have indicated $\eta_{\chi \chi}=\eta_{\chi}$ and $\xi_{\chi \chi \chi}^{2}=\xi_{\chi}^{2}$. Expanding out the slow-roll parameters in terms of potential derivatives, one finds that $L \simeq W_{, \chi} W_{, \chi \chi \chi} / 2 W_{, \chi \chi}^{2}$. If one considers a general power-law potential $V(\chi)=V_{0} \chi^{n}$ then $L=0$ for $n=0,1,2$ and $L \leq 1 / 2$ for $n \geq 3$. On the other hand, for an exponential potential $V(\chi)=V_{0} e^{\lambda \chi}$ then $L=1 / 2$. In these two cases we would therefore not expect to find a deviation from Eq. (4.5) beyond a factor of 2 . It is interesting to note that a potential of the form $V(\chi)=a \ln (\chi-b)$ for constants $a$ and $b$ has $L=1$ and so for such a potential the two leading order terms in $g_{\mathrm{NL}}$ exactly cancel and so $g_{\mathrm{NL}}$ is of order $f_{\mathrm{NL}} \times \mathcal{O}\left(\epsilon^{*}\right)$.

We now ask if there is a potential with $L \gg 1$. Considering a polynomial potential $V(\chi)$, the necessary condition is for the potential to possess a linear term, a negligible quadratic term and at least one term beyond quadratic order. The simplest such potential is a sloping inflection point of the form $V(\chi)=V_{0}+h \chi+\frac{1}{6} \lambda \chi^{3}$. Nearby the inflection point one has $\eta_{\chi} \simeq 0$ whilst $\theta \xi_{\chi}^{2}=M_{\mathrm{pl}}^{3} h \lambda / W_{0}^{2}$ and so $L$ diverges. We shall examine such an inflection point further in $\S 6$.

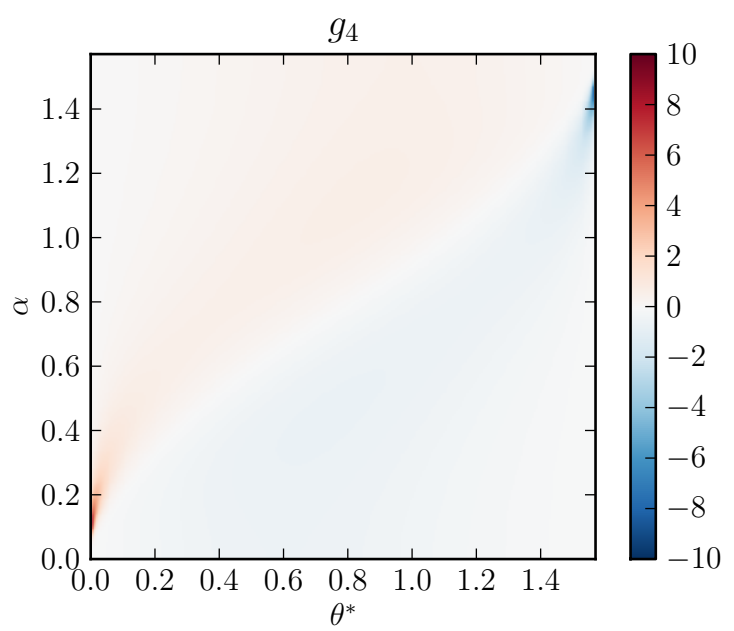

Figure 4. Heatmap of the function $g_{4}$ which is appropriately antisymmetric about field exchange. The very small lobe in the bottom left hand corner has positive $g_{4}$, whilst the lobe in the top right hand corner has negative $g_{4}$. This pattern is repeated for $g_{1}$ and $g_{3}$ as seen in Fig. 5 .

The $\Omega \neq 0$ case: It is considerably harder to make concrete statements about the value of the trispectrum parameter $g_{\mathrm{NL}}$, and its relation to $\tau_{\mathrm{NL}}$, when $\Omega \neq 0$. We can take a step 
in this direction by plotting the heatmaps for the remaining functions which appear in the expressions (3.17) and (3.18), $g_{1}$ and $g_{3}$. These are shown in Fig. 5. The similarity between these plots, and those of $f$ and $\tau$, tells us that the types of inflationary potential, and initial conditions, that can give rise to a large $g_{\mathrm{NL}}$ are similar to those that may give rise to a large $f_{\mathrm{NL}}$ and $\tau_{\mathrm{NL}}$. No new regions of interest appear for $g_{\mathrm{NL}}$ which were not present before.
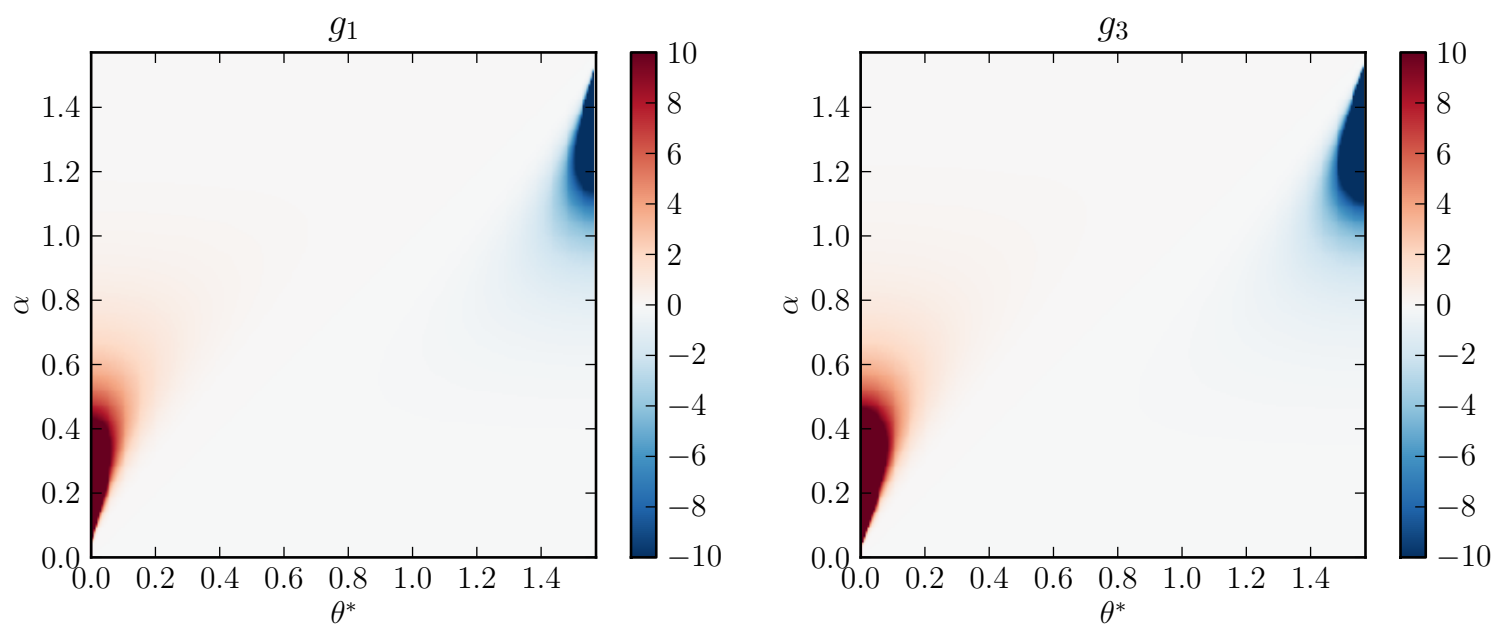

Figure 5. Plots of $g_{1}$ and $g_{3}$ on the same scale as used for the other heatmaps. We note that they are very similar and are anti-symmetric under field interchange. Both are large in the same regions of the parameter space as $\tau$.

The $\Omega \simeq 1$ case: Finally, we consider the case $\Omega \simeq 1$. This arises for the vacuum dominated potentials, and so can be relevant for models where inflation is terminated suddenly, perhaps through a waterfall transition. During a vacuum dominated phase of evolution, the fields evolve only very slowly, and likewise turns in field space progress slowly.

It is instructive to consider how the non-Gaussianity parameters given by Eqs. (3.8), (3.16) and (3.18) may be approximated for $\Omega \simeq 1, \theta \ll 1$ and a power-law potential of the form $V(\chi) \propto \chi^{n}$ where we presume $n$ to be a positive integer greater than or equal to 2 . In the regime of interest where $\theta \gg \theta^{*}$ (such that the functions $f$ and $\tau$ may be large) we require $|\chi| \gg\left|\chi^{*}\right|$. If $n=2$, such that the potential has a parabolic shape, then $\eta_{s s}$ is approximately constant and from Eq. (3.18) for $g_{\mathrm{NL}}$ we see that the first term will be negligible in this case. Furthermore, if the potential is well-described by an expansion to quadratic order we see that the other terms in $g_{\mathrm{NL}}$ are also suppressed with respect to $f_{\mathrm{NL}}$ and so $g_{\mathrm{NL}}$ is not large. This was the case found by Byrnes et al. [49] in their study of vacuum dominated quadratic potentials.

However, for $n \geq 3$ the situation is different. The isocurvature mass $\eta_{s s}$ is now a function of $\chi$ and $\left|\eta_{s s}\right|$ will grow as $|\chi|$ grows. In the regime of interest where $|\chi| \gg\left|\chi^{*}\right|$ we find that $\frac{54}{50} g_{\mathrm{NL}} \simeq \frac{1}{2} \tau_{\mathrm{NL}}$ from the first term in Eq. (3.18) and so it is clear that $g_{\mathrm{NL}}$ is not generally a vanishing quantity when the potential is described by terms beyond quadratic order. We note that the remaining terms in Eq. (3.18) for $g_{\mathrm{NL}}$ will mostly be small in this limit, but we will anticipate that the $g_{1} \xi_{s s s}^{2}$ term will contribute notably to the value of $g_{\mathrm{NL}}$ during this transient evolution. We shall look at the specific case of $n=3$ in $\S 5.3$. 


\section{Typical shapes in the inflationary potential}

In this section we briefly show how the formulae that we have found for a large $f_{\mathrm{NL}}, \tau_{\mathrm{NL}}$ and $g_{\mathrm{NL}}$ can be used to make quantitative predictions in certain generalised settings. We do this by studying how the bi- and tri-spectra transiently evolve as the field space trajectory passes over particular features in the inflationary potential. Motivated by the analyses in the previous sections, as well as other work [26, 39, 49], we consider potentials with one of the following three general features:

- a ridge,

- a valley,

- an inflection point.

Our analysis leads to simple expressions which give the sign and the peak values of the observable parameters generated by these features. By Taylor expanding a real potential about the initial conditions corresponding to a given inflationary potential, it is often possible to approximate the actual potential by one of these features over a range of field values. This can allow quantitative information about the predictions of models of inflation to be obtained readily without the need for detailed calculations [38].

The analyses of heatmaps show that large non-Gaussianity parameters require small angles in the parameter space which correspond to potentials for which the motion is highly aligned to one of the field directions, which we take to be $\phi$ without loss of generality. We can then expand the functions such as $f$ in the limit where $\theta^{*}$ and $\alpha$ are small to find

$$
f \simeq \frac{\theta^{6}}{\left(\theta^{4}+\theta_{*}^{2}\right)^{2}}, \quad \tau \simeq \frac{\theta^{8}}{\left(\theta^{4}+\theta_{*}^{2}\right)^{3}},
$$

and the other parameters are related as $g_{1} \simeq \theta \tau, 2 g_{3} \simeq \tau$ and $2 g_{4} \simeq \theta^{*} \tau$. We now consider each feature in turn.

\subsection{Potentials with a ridge}

The simplest possible ridge we can consider takes the form

$$
W=W_{0}+g \phi-\frac{1}{2} m_{\chi}^{2} \chi^{2},
$$

where $g$ and $m_{\chi}$ are taken to be positive and the $W_{0}$ term dominates. Since the potential is vacuum dominated and $\Omega \simeq 1$, the product-separable and sum-separable formulae for $f_{\mathrm{NL}}, \tau_{\mathrm{NL}}$ and $g_{\mathrm{NL}}$ are identical, because vacuum-domination also implies that $\epsilon \ll \eta_{s s}$. To be consistent with our sign convention we stipulate that $\chi<0$ such that $W_{, i}>0$ and $0 \leq \theta \leq \pi / 2$. The initial conditions are fine-tuned such that the field initially moves almost parallel to the top of the ridge, with $\theta \gtrsim 0$. The isocurvature direction is therefore almost precisely the $\chi$ direction, and $\eta_{s s} \simeq-m_{\chi}^{2} / W_{0}$ is a constant. This then gives

$$
\frac{6}{5} f_{\mathrm{NL}} \simeq f \eta_{\chi}^{*}, \quad \tau_{\mathrm{NL}} \simeq \tau \eta_{\chi}^{* 2}, \quad \frac{54}{50} g_{\mathrm{NL}} \simeq \frac{18}{5} f_{\mathrm{NL}} \eta_{\chi}^{*} .
$$

In this case $g_{\mathrm{NL}}$ is subdominant, even to $f_{\mathrm{NL}}$, and so we do not consider this further. The peak value of $f_{\mathrm{NL}}$ and $\tau_{\mathrm{NL}}$ can then be found by maximising the functions $f$ and $\tau$ as $\theta$ varies. 
They peak when $\theta^{2}=\sqrt{3} \theta^{*}$ and $\theta^{2}=\sqrt{2} \theta^{*}$ respectively and so

$$
\left.\frac{6}{5} f_{\mathrm{NL}}\right|_{\max } \simeq \frac{3 \sqrt{3}}{16} \frac{\eta_{\chi}^{*}}{\theta^{*}},\left.\quad \tau_{\mathrm{NL}}\right|_{\max } \simeq \frac{4}{27} \frac{\eta_{\chi}^{* 2}}{\theta_{*}^{2}} .
$$

The sign of $f_{\mathrm{NL}}$ is negative due to the sign of $\eta_{\chi}^{*}$, and the amplitude of $f_{\mathrm{NL}}$ reaches its peak after $\tau_{\mathrm{NL}}$. The peak of $\tau_{\mathrm{NL}}$ is only slightly larger than the square of $\left.\frac{6}{5} f_{\mathrm{NL}}\right|_{\mathrm{max}}$, but due to the difference in peaking times it is quite possible to have $\tau_{\mathrm{NL}} \gg\left|f_{\mathrm{NL}}\right|$.

\subsection{Potentials with a valley}

The simplest possible vacuum-dominated valley takes the form

$$
W=W_{0}+\frac{1}{2} m_{\phi}^{2} \phi^{2}+\frac{1}{2} m_{\chi}^{2} \chi^{2},
$$

where $m_{\phi}$ and $m_{\chi}$ are taken to be positive and the $W_{0}$ term dominates. We again consider $\theta * \ll 1$, and assume that $m_{\phi} \gg m_{\chi}$, so that the initial motion is almost exactly parallel to the $\phi$ direction. Once again, therefore, the isocurvature direction is well approximated by the $\chi$ direction, and $\eta_{s s}=m_{\chi}^{2} / W_{0}$ is again constant. This then gives expressions for $f_{\mathrm{NL}}$, $\tau_{\mathrm{NL}}$ and $g_{\mathrm{NL}}$ which are identical to those in Eq. (5.3), but we note that the signs of $f_{\mathrm{NL}}$ and $g_{\mathrm{NL}}$ are now reversed.

\subsection{Potentials with an inflection point}

The analysis of $\S 4.2 .2$ with $\Omega \simeq 1$ illustrated how $g_{\mathrm{NL}}$ is negligible for inflationary potentials that are approximately quadratic, but that $g_{\mathrm{NL}} \sim \tau_{\mathrm{NL}}$ for potentials with cubic or higher order shapes. We now consider the simplest such shape, with an inflection point of the form

$$
W=W_{0}+g \phi+\frac{1}{6} \lambda \chi^{3} .
$$

The regime of interest is close to but below the inflection point with $|\chi| \ll 1$ such that $\theta \ll 1$. The non-Gaussianity parameters are only large in the regimes where $f$ or $\tau$ are large, which requires $|\chi| \gg\left|\chi^{*}\right|$. In this limit we find that the leading order non-Gaussianity is given as

$$
\frac{6}{5} f_{\mathrm{NL}} \simeq 2 f \eta_{s s}, \quad \tau_{\mathrm{NL}} \simeq 4 \tau \eta_{s s}^{2}, \quad \frac{54}{50} g_{\mathrm{NL}} \simeq \frac{5}{8} \tau_{\mathrm{NL}} .
$$

For a general power-law potential, the numerical relation between $g_{\mathrm{NL}}$ and $\tau_{\mathrm{NL}}$ will take a different value. We note that $g_{\mathrm{NL}}$ simply follows the evolution of $\tau_{\mathrm{NL}}$ and so we need only calculate the peak values of $f_{\mathrm{NL}}$ and $\tau_{\mathrm{NL}}$. We find

$$
\left.\frac{6}{5} f_{\mathrm{NL}}\right|_{\max } \simeq-1.08 \frac{\left|\xi_{s s s}^{*}\right|}{\theta^{* 3 / 4}},\left.\quad \tau_{\mathrm{NL}}\right|_{\max } \simeq 1.48 \frac{\xi_{s s s}^{* 2}}{\theta^{* 3 / 2}} .
$$

where these peaks respectively occur when $\theta^{2}=\sqrt{3} \theta^{*}$ and $\theta^{2}=\sqrt{13 / 3} \theta^{*}$.

\section{Concrete Models}

In the preceding discussion we developed a number of tools for analysing a given two-field inflationary model. In this section we demonstrate the usefulness of these tools, by showing concrete examples of simple models which can produce large values of $\tau_{\mathrm{NL}}$ and/or $g_{\mathrm{NL}}$. 


\subsection{Two-field hybrid inflation}

The original hybrid model was introduced by Linde [59] as an effectively single field model of inflation, where the job of ending inflation was assigned to a second field, known as the waterfall field. The interesting feature of this set up is that inflation does not end gracefully, but rather through a sudden transition, which allows more freedom in constructing the potential that drives inflation. This model has been extended to the multi-field case, where two or more scalar fields drives inflation before the waterfall field ends it. In this section we consider a two-field hybrid model of inflation with the potential [28]

$$
W=W_{0}\left(1+\frac{1}{2} \eta_{\phi} \phi^{2}+\frac{1}{2} \eta_{\chi} \chi^{2}\right)-\left(f \phi^{2}+g \phi \chi+h \chi^{2}\right) \frac{\psi^{2}}{2}+\frac{1}{2} m_{\psi}^{2} \psi^{2},
$$

where the waterfall field $\psi$ is held at 0 during inflation, rolling rapidly to it's true minimum when

$$
m_{\psi}^{2}=f \phi^{2}+g \phi \chi+h \chi^{2} .
$$

Since inflation ends suddenly in this model before isocurvature modes decay, we need to account for the possibility that inflation might end on a surface other than one of constant energy density. This implies that the curvature perturbation produced by this model can be split into two parts,

$$
\zeta=\zeta_{\text {inf }}+\zeta_{\mathrm{e}}
$$

where $\zeta_{\text {inf }}$ is the part generated during inflation, and $\zeta_{e}$ is the part generated from the end of inflation dynamics. The condition (6.2) for the end of inflation also defines the surface on which inflation ends, which in this case is an ellipse. Varying $f, g$ and $h$ determines the orientation and the eccentricity of the ellipse in the $\{\phi, \chi\}$ space; with $g=0$ defining an ellipse aligned with the axes, and $h=f$ with $g=0$ defining a circle. With this in mind, to determine which contribution in Eq. (6.3) dominates we look at the relationship between $f, h$ and $g$. For $g \gg f, h$ the $\zeta_{\mathrm{e}}$ term dominates over $\zeta_{\text {inf }}[60,61]$. We consider this case in the following subsection, and in the remainder of this section focus on the scenario where $\zeta \simeq \zeta_{\text {inf }}$ where the spectrum, bispectrum and trispectrum will evolve as described in our previous analysis for a two-field model, and there is no significant additional contribution to $\zeta$ at the end of inflation. The characteristics and various observational predictions of this model have been studied extensively in the literature, see for example Refs. [26, 27, 38, 39, 49, 52, 57, 62-69].

We restrict our attention to the case in which $\eta_{\phi}$ and $\eta_{\chi}$ are both positive, and so the potential during inflation has a valley-like shape. This provides an illustrative example of our discussion in $\S 5.2$. If either $\eta_{\phi}$ or $\eta_{\chi}$ were negative, the potential would contain a ridge. We further assume that $\eta_{\phi}>\eta_{\chi}$, and that the initial conditions are such that $0<\chi^{*} \ll M_{\mathrm{pl}}$, whilst $\phi^{*} \sim \mathcal{O}\left(M_{\mathrm{pl}}\right)$ is significantly displaced from zero. Hence, the trajectory initially rolls towards the minimum along the $\phi$ axis before turning and rolling very slowly towards the $\chi$ axis.

These initial conditions imply a small $\theta^{*}$, and since the potential is vacuum-dominated, $\alpha \simeq \theta$ and the heatmaps are particularly simple to interpret. The initial (horizon crossing) condition corresponds to a position on the diagonal in the bottom left hand corner of any given heatmap. As the trajectory begins its slow turn into the valley, the point on the heat-map proceeds vertically upwards and we can see that a strong non-Gaussian signal is expected during the early stages of the turn. When the angle grows larger, the trajectory moves out of the regions of the heatmaps where $f$ and $\tau$ are large and so the non-Gaussianity 


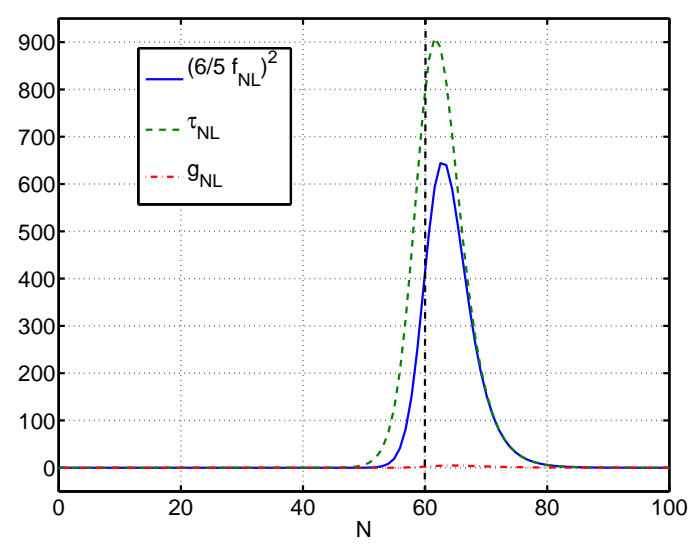

Figure 6. Evolution of $\operatorname{sign}\left(f_{\mathrm{NL}}\right)\left(6 / 5 f_{\mathrm{NL}}\right)^{2}$ (solid blue line), $\tau_{\mathrm{NL}}$ (dashed green line), and $g_{\mathrm{NL}}$ (dotdashed red line) for the two-field hybrid model described in the text, calculated using the analytic formulae presented in the appendix. The vertical dashed line at 60 efolds indicates where we choose the waterfall field to terminate inflation. We also continue the evolution past this point, as if the waterfall field were absent, to show the subsequent peak and decay of the non-Gaussianity.

decays. Since the large non-Gaussianity occurs during the early stages of the turn we see that $\eta_{s s} \approx \eta_{\chi}$ is a constant whilst all components of the $\xi_{i j k}^{2}$ parameter are zero.

In Fig. 6 we show the evolution of $f_{\mathrm{NL}}, \tau_{\mathrm{NL}}$ and $g_{\mathrm{NL}}$ for the potential (6.1). The parameter values used were $\eta_{\phi}=0.09$ and $\eta_{\chi}=0.0025$ with the initial conditions taken as $\phi^{*}=0.9 M_{\mathrm{pl}}$ and $\chi^{*}=0.001 M_{\mathrm{pl}}$. The evolution of the trispectrum is exactly as we expect: $f_{\mathrm{NL}}$ and $\tau_{\mathrm{NL}}$ both begin at negligible values, grow to large positive peaks and then decay again to negligible values. We confirm that the peak amplitudes are as expected from Eq. (5.4) in $\S 5.2$ to within $10 \%$. Furthermore, we see that $\tau_{\mathrm{NL}}$ peaks before $f_{\mathrm{NL}}$ in agreement with the discussion regarding Eq. (3.16) and also in agreement with the results in $\S 5.2$. This quadratic shape of the potential gives us a small $g_{\mathrm{NL}}$ as discussed in $\S 4.2 .2$.

After 60 efolds, where we assume the evolution is terminated by the waterfall field, we find $n_{s}=1.07$, which is clearly in violation of the bounds on the spectral index placed by WMAP, which demands a red spectral tilt for priors of zero running and tensor to scalar ratio. As this model predicts neither a significant tensor to scalar ratio $\left(\gtrsim 10^{-2}\right)$ nor a large negative running, we do not consider this model a viable candidate for inflation. Moreover, we know that there does not exist a choice of parameters or initial conditions (where both fields are less than the Planck scale) which can support a red tilt [26]. Nevertheless, we still consider the model a useful illustration of our analysis, and in particular of the a potential with a valley feature. It is also of note that a full treatment of the hybrid model may lead to the production of cosmic strings (for example see Refs. [70, 71]) and if cosmic strings were to contribute fractionally to the temperature power spectrum, then the best fit parameters would support a blue spectral tilt (see for example Refs. [72, 73]).

\subsubsection{The role of the end of inflation hypersurface}

As we have described, in addition to allowing for interesting phase space evolution during inflation, multi-field models of inflation can also have an end of inflation surface different from one of uniform energy density [57,60,61, 68, 74, 75], and this leads to the the extra contribution to the curvature perturbation $\zeta_{\mathrm{e}}$ discussed above. If this extra contribution 
dominates then the overall curvature perturbation can be significantly non-Gaussian and so the trispectrum may be enhanced.

Here we do not attempt a full analysis of the statistics when $\zeta_{\mathrm{e}}$ is accounted for, but rather offer one simple example. We assume $\zeta_{\mathrm{e}} \gg \zeta_{\text {inf }}$, and consider the case where $\chi$ is held at zero during inflation and the parameters $\eta_{\phi}$ and $\eta_{\chi}$ are equal. The slow-roll parameters in this case are given as [60]

$$
\eta_{s s}=\eta_{\chi}=\eta_{\phi}, \quad \epsilon=\frac{\eta_{s s}^{2}}{2} \phi^{2}
$$

and $g_{\mathrm{NL}}$ and $\tau_{\mathrm{NL}}$ parameters at the end of inflation are found to be

$$
\begin{aligned}
g_{\mathrm{NL}} & \simeq \frac{50 g}{54}\left(3 f_{\mathrm{NL}}\left(\eta_{s s}-2 \epsilon\right)+\eta_{s s}\right), \\
\tau_{\mathrm{NL}} & \simeq 2 \epsilon-\eta_{s s}+\frac{12 f_{\mathrm{NL}}}{5}\left(\frac{9 f_{\mathrm{NL}}}{10}-1\right), \\
f_{\mathrm{NL}} & =\frac{5 f \eta_{s s}}{3 g}\left(\frac{2 h}{g}-\frac{g}{2 f}\right),
\end{aligned}
$$

where all quantities are evaluated at the end of inflation.

We see that $\tau_{\mathrm{NL}}$ closely follows the single-field result $\tau_{\mathrm{NL}} \simeq\left(6 f_{\mathrm{NL}} / 5\right)^{2}$ and so may be detectable if $f_{\mathrm{NL}}$ is observably large as found in ref. [60]. When $f_{\mathrm{NL}} \gg 1$, we see that $g_{\mathrm{NL}} \propto g f_{\mathrm{NL}} \times \mathcal{O}(\epsilon)$ and when $f_{\mathrm{NL}} \ll 1$ then $g_{\mathrm{NL}} \propto g \times \mathcal{O}(\epsilon)$. In both cases $g_{\mathrm{NL}}$ is negligible unless one considers a very large coupling $g$ that is inconsistent with effective field theory and so we see that, for this particular model, modifying the end of inflation hypersurface produces non-Gaussianitites that are accounted for purely by the bispectrum.

In Ref. [64], the author relates the non-Gaussianity predictions from the end of inflation hypersurface to the geometry of this surface. The author finds $g_{\mathrm{NL}}$ may be significant when the curvature changes rapidly along the surface. Our findings here agree with this conclusion, because the parameter choices $(g \gg 1)$ which lead to an enhanced $g_{\mathrm{NL}}$ correspond to a large ellipticity and have the trajectory lying very close to the semi-major axis.

\subsection{Axion Potential}

The model with the potential

$$
W=\frac{1}{4} g \phi^{4}+\Lambda^{4}(1-\cos (2 \pi \chi / f)),
$$

is inspired by the N-axion model studied in Refs. [51, 53]. It is similar to the model introduced in Ref. [38] as an example of a two-field potential which has an interesting transitory evolution as well as a large asymptotic value of non-Gaussianity at the adiabatic limit. Here we have chosen the $\phi$ field to self-interact through a quartic potential, which will allow a choice of parameters and initial conditions for which $\zeta$ can become constant during the slow-roll evolution, while still giving a value of the spectral index consistent with observations ${ }^{5}$. The potential (6.6) contains an infinite number of successive ridges and valleys. Taking initial

\footnotetext{
${ }^{5}$ In Ref. [39], an example was given in which a large non-Gaussianity was reached during slow-roll inflation. We note, however, that the spectral index of this example was much too low to be consistent with observation. By choosing a quartic potential in this work, our aim is to show that there is no barrier in principal to having a model which reaches the adiabatic limit during slow-roll inflation with a consistent value of $n_{s}$.
} 
conditions such that $\chi^{*}$ is sufficiently close to $f / 2$, while $\phi$ starts its evolution at a large field value ( $\phi^{*} \approx 23 M_{\mathrm{pl}}$ is required for roughly 60 efolds), the trajectory is initially almost entirely in the $\phi$ direction and rolls along the ridge defined by $\chi=f / 2$. The initial angle $\theta^{*}$ is therefore close to zero, and as the trajectory slowly turns off the ridge, $\theta$ increases.

From the the heatmaps, one can see that the initial growth of the angle $\theta$ will lead to an increase in the magnitude of $f_{\mathrm{NL}}$ and $\tau_{\mathrm{NL}}$ as $\alpha$ passes up through the 'hot-spot' region where $f_{\mathrm{NL}}$ and $\tau_{\mathrm{NL}}$ peak. Since the third derivative of the potential is insignificant in the early stages of this turn, $g_{\mathrm{NL}}$ is not significant. After the initial turn from the ridge-top, the trajectory will fall quickly down the steep side of the ridge, before turning back towards its original direction as it reaches the valley bottom. This leads to a decrease of $\alpha$ back through the hot-spot region once more. Importantly, the potential $W$ drops by a small (but non-vanishing) amount between these two turns. From Eq.(4.2) this means that the initial growth of $\alpha$ is slightly larger than the subsequent decay and so $\alpha$ will not make it to zero as $\theta \rightarrow 0$. Instead, it will end up with a small positive value. We therefore expect the nonGaussianity parameters to take large constant values asymptotically, where we also know that the phase space trajectory ends up in a valley and so the asymptotic formulae for $g_{\mathrm{NL}}$ in Eq. (4.5) will be an accurate prediction.

In Fig. 7 we give the evolution of $f_{\mathrm{NL}}, \tau_{\mathrm{NL}}$ and $g_{\mathrm{NL}}$ for a specific realisation of this case described above. The parameter values are $f=1 M_{\mathrm{pl}}$ and $\Lambda^{4} / g=(25 / 2 \pi)^{2} M_{\mathrm{pl}}^{4}$, with the overall normalisation fixed to agree with WMAP7 power spectrum amplitude. The initial conditions are $\phi^{*}=22.5 M_{\mathrm{pl}}$ and $\chi^{*}=f / 2-0.001 M_{\mathrm{pl}}$ which gives us the HCA value of $\alpha$ as 0.022 which is small in agreement with the above discussion. In this case, the final constant spectral index has a value of $n_{s}=0.949$ which is within the WMAP7 $95 \%$ contours. The evolution is exactly as we expect, with $f_{\mathrm{NL}}$ and $\tau_{\mathrm{NL}}$ both beginning with negligible values and $f_{\mathrm{NL}}$ then evolving to a large negative peak while $\tau_{\mathrm{NL}}$ grows to a large positive one, before both peaks decay. Despite the potential dropping quite significantly before the axion rolls, the peak values of $f_{\mathrm{NL}}$ and $\tau_{\mathrm{NL}}$ are described by the formulae in $\S 5.1$ with about $30 \%$ accuracy. The subsequent evolution is also interesting. As the trajectory evolves into the valley there is a positive spike in $f_{\mathrm{NL}}$ and $g_{\mathrm{NL}}$ typical of this evolution. Furthermore, $g_{\mathrm{NL}}$ also increases significantly as we move away from the $\Omega=1$ regime and we see that there is a delay between the evolution of $f_{\mathrm{NL}}$ and $\tau_{\mathrm{NL}}$ as discussed in $\S 5.1$. Finally, the constant asymptotic values of $f_{\mathrm{NL}}, \tau_{\mathrm{NL}}$ and $g_{\mathrm{NL}}$ are reached where we find Eq. (4.5) holds very well. In this case $\tau_{\mathrm{NL}} \approx\left(6 / 5 f_{\mathrm{NL}}\right)^{2}$, though this is not a necessary consequence of reaching the asymptotic regime, unlike the relation between $\tau_{\mathrm{NL}}$ and $g_{\mathrm{NL}}$.

\subsection{Inflection point model}

The discussion in $\S 4.2 .2$ motivated sloping inflection features in the potential as a scenario in which the condition $g_{\mathrm{NL}} \gg \tau_{\mathrm{NL}}$ might be found in an adiabatic regime where $\Omega \rightarrow 0$. We now present such a model, but note that the tuning required to find parameters such that $g_{\mathrm{NL}}$ is both large and dominant in the adiabatic limit is severe. We expect, therefore, that the simple relation (4.5) will hold for the vast majority of models.

We choose an inflection potential similar to that studied in Ref. [39] which takes the form

$$
W=W_{0}\left(\frac{1}{4 !} \phi^{4}+V_{0}+h \chi+\frac{1}{3 !} \lambda \chi^{3}+\frac{1}{4 !} \mu \chi^{4}\right) .
$$

The value of $W_{0}$ is fixed by the WMAP power spectrum, and there is an inflection point feature at $\chi=0$. We further assume that $V_{0}$ and $\mu$ are fixed by the requirement that there 


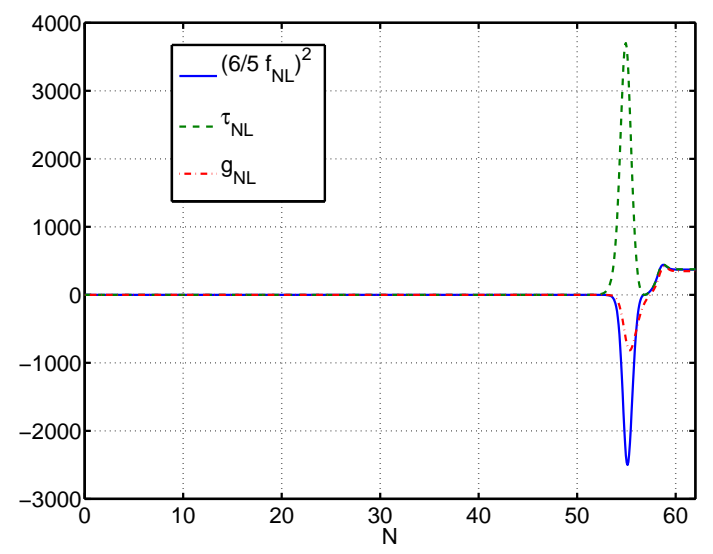

Figure 7. Evolution of $\operatorname{sign}\left(f_{\mathrm{NL}}\right)\left(6 / 5 f_{\mathrm{NL}}\right)^{2}$ (solid blue line), $\tau_{\mathrm{NL}}$ (dashed green line), and $g_{\mathrm{NL}}$ (dotdashed red line) for the axion model discussed in the text, calculated using the analytic formulae presented in the appendix.

is a minimum of the potential at $\chi_{\min }=-\rho$, where $\rho$ is taken to be positive. We note that beginning some way above/below the inflection point leads to a negative/positive asymptotic value of $f_{\mathrm{NL}}$ as explored in Ref. [39].

From an exploration of the parameter space for this model we have found it very difficult to obtain a large and dominant $g_{\mathrm{NL}}$ whilst satisfying the constraints of slow-roll. We present a compromise scenario where the $\chi$ field rolls when $\epsilon \sim 0.3$. Such a model has parameter values $h=0.05 M_{\mathrm{pl}}^{3}, \lambda=10^{4} M_{\mathrm{pl}}$ and $\rho=0.1 M_{\mathrm{pl}}$ and the evolution begins with $\phi^{*}=22.5 M_{\mathrm{pl}}$ and $\chi^{*}=0{ }^{6}$. We compare the evolution from our analytic expressions to the numerical non-slow-roll evolution calculated using a finite-difference code identical to that used in Ref [38]. In the non-slow-roll case, the scalar field velocity perturbations decouple from the field perturbations and so the phase space of the dynamics is enlarged. We see the effect of this by the presence of oscillatory behaviour in the non-Gaussianity parameters and in the spectral index. When the deviation from slow-roll is not too large, the non-slow-roll evolution mimics a superposition of the the slow-roll evolution and these rapid oscillations. We therefore find that our analytic results are surprisingly applicable when slow-roll begins to break. For this example, the analytic spectral index is $n_{s}=0.964$ and this is within $1 \%$ of the non-slow-roll value. Our calculation gives $g_{\mathrm{NL}}=-432$ which agrees with the non-slow-roll code to within a factor of two. In general, if the evolution of the observables for a given model have not settled down to a constant value before the end of slow-roll inflation then one must use numerical methods to obtain an accurate result. However, a crude estimate is very easy to calculate through the slow-roll analysis. To conclude, in this final example we have tried to engineer a model to break from the simple $g_{\mathrm{NL}}-\tau_{\mathrm{NL}}$ result in Eq. (4.5) but we have found that this is very hard to achieve.

\footnotetext{
${ }^{6}$ A realistic application of our formulae requires that the model is insensitive to changes in the horizon crossing conditions within the range of the quantum scatter. Such changes do not affect the predictions of this model.
} 


\section{Conclusions}

In this work we have studied non-Gaussianities in two-field slow-roll inflation. By extending and simplifying the 'heatmap' analysis of Byrnes et al. [27] we have shown, for separable potentials, the regions of the parameter space that can give rise to large non-Gaussianities, both in the bi- and tri-spectra. This analysis also benefits from showing the explicit dependence of these non-Gaussianites on the shape of the inflationary potential, and the Horizon Crossing Approximation can be easily applied to our results. The analyses in the paper allows us to make the following conclusions:

- We have found that the regions of the heatmap parameter space that produce large values of the bispectrum parameter $f_{\mathrm{NL}}$ are also capable of producing large values of the trispectrum parameters $\tau_{\mathrm{NL}}$ or $g_{\mathrm{NL}}$. Our results confirm that a necessary requirement for a large local non-Gaussianity is that the horizon crossing field velocities must be dominated by one of the two fields.

- The relationship between $\tau_{\mathrm{NL}}$ and $f_{\mathrm{NL}}$ is shown explicitly for separable potentials and is given in Eq. (3.16).

- We have found that in the adiabatic limit the non-Gaussianity parameters $g_{\mathrm{NL}}$ and $\tau_{\mathrm{NL}}$ can be related by Eq. (4.5), which is confirmed for a variety of models considered here. This relation can be modified when the isocurvature third slow-roll parameter $\xi_{s s s}^{2}$ is significant.

- We have shown that the ridge and valley features in the inflationary potential can lead to large $\tau_{\mathrm{NL}}$ and $f_{\mathrm{NL}}$ parameters, with $\tau_{\mathrm{NL}}$ being the first to peak. $g_{\mathrm{NL}}$ can be large if the potential is described by terms beyond quadratic order. It is possible, but very hard to engineer, that $g_{\mathrm{NL}}$ is the dominant statistic if inflation reaches a natural adiabatic limit.

- We have employed the heatmaps in a dynamic way, and shown that they can be used as qualitative tools, in order to understand and predict the evolution of non-Gaussianities in different models of inflation.

Forthcoming Planck data will further constrain primordial non-Gaussianities. Since our analysis relates these observations to the shape of the multi-field inflationary potential, it may be that if a signal is detected one could infer information about the inflationary potential from observation. This is an exciting possibility. Moreover, the non-Gaussianity parameters considered here are not the only parameters that can act as signatures for specific shapes in the potential. In particular, it would be valuable to extend our analysis to consider the running of the spectral index and the running of $f_{\mathrm{NL}}, g_{\mathrm{NL}}$ and $\tau_{\mathrm{NL}}$, which we intend to return to in future work.

\section{Acknowledgements}

The authors would like to thank David Seery and Chris Byrnes for helpful discussions. JE is supported by a Science and Technology Facilities Council studentship. LA is supported by the Japanese Society for the Promotion of Science (JSPS). IH, DJM and RT are supported by Science and Technology Facilities Council grant ST/J001546/1. 


\section{A Summary of results for two-field $\delta N$ calculations.}

\section{A.1 Sum-separable potentials}

This section follows from the work of Vernizzi and Wands [25] and considers a potential with the sum-separable form $W=U(\phi)+V(\chi)$. The first derivatives of $N$ are

$$
\begin{array}{ll}
M_{\mathrm{pl}} N_{, \phi}=\frac{u}{\sqrt{2 \epsilon_{\phi}^{*}}}, & u=\frac{U^{*}+Z}{W^{*}}, \\
M_{\mathrm{pl}} N_{, \chi}=\frac{v}{\sqrt{2 \epsilon_{\chi}^{*}}}, & v=\frac{V^{*}-Z}{W^{*}},
\end{array}
$$

where

$$
Z=\frac{V \epsilon_{\phi}-U \epsilon_{\chi}}{\epsilon} .
$$

For notational ease we have dropped the ' $c$ ' label that is commonly attached to quantities evaluated on a later-time uniform density hypersurface. For the purposes of calculating the trispectrum we shall also need to decompose $\eta_{i j}$ and $\xi_{i j k}^{2}$ into the kinematic basis on the uniform density hypersurface. In the original $\{\phi, \chi\}$ frame the potential is sum-separable and the only non-zero values of $\eta_{i j}$ and $\xi_{i j k}^{2}$ are those for which all of the indices are identical, which allows us to use the single-index notation $\eta_{\phi}$ and $\xi_{\phi}^{2}$. After rotating into the kinematic basis with fields $\sigma$ and $s$, the potential generally loses it separable form and so it is necessary to use all of the indices. The three $\eta$ components are

$$
\begin{aligned}
& \eta_{\sigma \sigma}=\frac{\epsilon_{\phi} \eta_{\phi}+\epsilon_{\chi} \eta_{\chi}}{\epsilon} \\
& \eta_{\sigma s}=\frac{\sqrt{\epsilon_{\phi} \epsilon_{\chi}}}{\epsilon}\left(\eta_{\chi}-\eta_{\phi}\right), \\
& \eta_{s s}=\frac{\epsilon_{\chi} \eta_{\phi}+\epsilon_{\phi} \eta_{\chi}}{\epsilon}
\end{aligned}
$$

The components of $\xi_{i j k}^{2}$ in the kinematic frame are

$$
\begin{aligned}
& \epsilon^{3 / 2} \xi_{\sigma \sigma \sigma}^{2}=\epsilon_{\chi}^{3 / 2} \xi_{\chi}^{2}+\epsilon_{\phi}^{3 / 2} \xi_{\phi}^{2}, \\
& \epsilon^{3 / 2} \xi_{\sigma \sigma s}^{2}=\epsilon_{\chi} \sqrt{\epsilon_{\phi}} \xi_{\chi}^{2}-\epsilon_{\phi} \sqrt{\epsilon_{\chi}} \xi_{\phi}^{2}, \\
& \epsilon^{3 / 2} \xi_{\sigma s s}^{2}=\epsilon_{\phi} \sqrt{\epsilon_{\chi}} \xi_{\chi}^{2}+\epsilon_{\chi} \sqrt{\epsilon_{\phi}} \xi_{\phi}^{2}, \\
& \epsilon^{3 / 2} \xi_{s s s}^{2}=\epsilon_{\phi}^{3 / 2} \xi_{\chi}^{2}-\epsilon_{\chi}^{3 / 2} \xi_{\phi}^{2} .
\end{aligned}
$$

The second derivatives of $N$ follow by differentiation of Eqs. (A.1), giving

$$
\begin{aligned}
& M_{\mathrm{pl}}^{2} N_{, \phi \phi}=1-\frac{u \eta_{\phi}^{*}}{2 \epsilon_{\phi}^{*}}+\frac{\mathcal{A}}{\epsilon_{\phi}^{*}}, \\
& M_{\mathrm{pl}}^{2} N_{, \phi \chi}=-\frac{\mathcal{A}}{\sqrt{\epsilon_{\phi}^{*} \epsilon_{\chi}^{*}}}, \\
& M_{\mathrm{pl}}^{2} N_{, \chi \chi}=1-\frac{v \eta_{\chi}^{*}}{2 \epsilon_{\chi}^{*}}+\frac{\mathcal{A}}{\epsilon_{\chi}^{*}},
\end{aligned}
$$


where

$$
\frac{M_{\mathrm{pl}}}{W^{*}} \sqrt{\frac{\epsilon_{\phi}^{*}}{2}} \frac{\partial Z}{\partial \phi^{*}}=-\frac{M_{\mathrm{pl}}}{W^{*}} \sqrt{\frac{\epsilon_{\chi}^{*}}{2}} \frac{\partial Z}{\partial \chi^{*}}=\mathcal{A} \equiv \frac{W^{2}}{W_{*}^{2}} \frac{\epsilon_{\phi} \epsilon_{\chi}}{\epsilon^{2}}\left(\eta_{s s}-\epsilon\right) .
$$

Taking the next derivative we find

$$
\begin{aligned}
& M_{\mathrm{pl}}^{3} N_{, \phi \phi \phi}=\frac{1}{\epsilon_{\phi}^{*} \sqrt{2 \epsilon_{\phi}^{*}}}\left(-\frac{u}{2} \sqrt{\frac{\epsilon_{\phi}^{*}}{\epsilon^{*}}} \xi_{\phi}^{* 2}-\epsilon_{\phi}^{*} \eta_{\phi}^{*}+u \eta_{\phi}^{* 2}-3 \eta_{\phi}^{*} \mathcal{A}+\mathcal{B}^{2}\right), \\
& M_{\mathrm{pl}}^{3} N_{, \phi \phi \chi}=\frac{1}{\epsilon_{\phi}^{*} \sqrt{2 \epsilon_{\chi}^{*}}}\left(\eta_{\phi}^{*} \mathcal{A}-\mathcal{B}^{2}\right), \\
& M_{\mathrm{pl}}^{3} N_{, \phi \chi \chi}=\frac{1}{\epsilon_{\chi}^{*} \sqrt{2 \epsilon_{\phi}^{*}}}\left(\eta_{\chi}^{*} \mathcal{A}+\mathcal{B}^{2}\right), \\
& M_{\mathrm{pl}}^{3} N_{, \chi \chi \chi}=\frac{1}{\epsilon_{\chi}^{*} \sqrt{2 \epsilon_{\chi}^{*}}}\left(-\frac{v}{2} \sqrt{\frac{\epsilon_{\chi}^{*}}{\epsilon^{*}}} \xi_{\chi}^{* 2}-\epsilon_{\chi}^{*} \eta_{\chi}^{*}+v \eta_{\chi}^{* 2}-3 \eta_{\chi}^{*} \mathcal{A}-\mathcal{B}^{2}\right),
\end{aligned}
$$

where

$$
\begin{aligned}
M_{\mathrm{pl}} \sqrt{2 \epsilon_{\phi}^{*}} \frac{\partial \mathcal{A}}{\partial \phi_{*}} & \equiv-4 \epsilon_{\phi}^{*} \mathcal{A}+\mathcal{B}^{2} \\
\mathcal{B}^{2} & \equiv-\frac{W^{3}}{W_{*}^{3}} \frac{{\sqrt{\epsilon_{\phi} \epsilon_{\chi}}}^{3}}{\epsilon^{3}}\left[\xi_{s s s}^{2}+2 \frac{\epsilon_{\phi}-\epsilon_{\chi}}{\sqrt{\epsilon_{\phi} \epsilon_{\chi}}} \eta_{s s}\left(\eta_{s s}-\epsilon\right)-2 \eta_{\sigma s}\left(\eta_{s s}+\epsilon\right)\right] .
\end{aligned}
$$

The other derivative of $\mathcal{A}$ follows by a simple permutation of Eq. (A.12) under the joint permutations $\{u \leftrightarrow v\}$ and $\{\phi \leftrightarrow \chi\}$ which has the effect of negating $\mathcal{B}^{2}$ whilst $\mathcal{A}$ remains unchanged. We take these results together to find

$$
\begin{aligned}
& n_{s}-1=-4\left(\frac{u^{2}}{\epsilon_{\phi}^{*}}+\frac{v^{2}}{\epsilon_{\chi}^{*}}\right)^{-1}\left[1-\frac{u^{2} \eta_{\phi}^{*}}{2 \epsilon_{\phi}^{*}}-\frac{v^{2} \eta_{\chi}^{*}}{2 \epsilon_{\chi}^{*}}\right]-2 \epsilon^{*} \\
& f_{\mathrm{NL}}=\frac{5}{6}\left(\frac{u^{2}}{\epsilon_{\phi}^{*}}+\frac{v^{2}}{\epsilon_{\chi}^{*}}\right)^{-2}\left[2\left(\frac{u^{2}}{\epsilon_{\phi}^{*}}+\frac{v^{2}}{\epsilon_{\chi}^{*}}\right)-\frac{u^{3} \eta_{\phi}^{*}}{\epsilon_{\phi}^{* 2}}-\frac{v^{3} \eta_{\chi}^{*}}{\epsilon_{\chi}^{* 2}}+2\left(\frac{u}{\epsilon_{\phi}^{*}}-\frac{v}{\epsilon_{\chi}^{*}}\right)^{2} \mathcal{A}\right], \\
& \tau_{\mathrm{NL}}=4\left(\frac{u^{2}}{\epsilon_{\phi}^{*}}+\frac{v^{2}}{\epsilon_{\chi}^{*}}\right)^{-3}\left[-\frac{u^{3} \eta_{\phi}^{*}}{\epsilon_{\phi}^{* 2}}-\frac{v^{3} \eta_{\chi}^{*}}{\epsilon_{\chi}^{* 2}}+\frac{u^{4} \eta_{\phi}^{* 2}}{4 \epsilon_{\phi}^{* 3}}+\frac{v^{4} \eta_{\chi}^{* 2}}{4 \epsilon_{\chi}^{* 3}}+\frac{u^{2}}{\epsilon_{\phi}^{*}}+\frac{v^{2}}{\epsilon_{\chi}^{*}}\right. \\
& -\frac{u^{2}}{\epsilon_{\phi}^{* 2}}\left(\frac{u}{\epsilon_{\phi}^{*}}-\frac{v}{\epsilon_{\chi}^{*}}\right) \eta_{\phi}^{*} \mathcal{A}-\frac{v^{2}}{\epsilon_{\chi}^{* 2}}\left(\frac{v}{\epsilon_{\chi}^{*}}-\frac{u}{\epsilon_{\phi}^{*}}\right) \eta_{\chi}^{*} \mathcal{A} \\
& \left.+2\left(\frac{u}{\epsilon_{\phi}^{*}}-\frac{v}{\epsilon_{\chi}^{*}}\right)^{2} \mathcal{A}+\left(\frac{u}{\epsilon_{\phi}^{*}}-\frac{v}{\epsilon_{\chi}^{*}}\right)^{2}\left(\frac{1}{\epsilon_{\phi}^{*}}+\frac{1}{\epsilon_{\chi}^{*}}\right) \mathcal{A}^{2}\right], \\
& g_{\mathrm{NL}}=\frac{50}{54}\left(\frac{u^{2}}{\epsilon_{\phi}^{*}}+\frac{v^{2}}{\epsilon_{\chi}^{*}}\right)^{-3}\left[-\frac{u^{3} \eta_{\phi}^{*}}{\epsilon_{\phi}^{* 2}}-\frac{v^{3} \eta_{\chi}^{*}}{\epsilon_{\chi}^{* 2}}+\frac{u^{4} \eta_{\phi}^{* 2}}{\epsilon_{\phi}^{* 3}}+\frac{v^{4} \eta_{\chi}^{* 2}}{\epsilon_{\chi}^{* 3}}-\frac{1}{2} \frac{u^{4} \xi_{\phi}^{* 2}}{\epsilon_{\phi}^{* 2} \sqrt{\epsilon^{*} \epsilon_{\phi}^{*}}}-\frac{1}{2} \frac{v^{4} \xi_{\chi}^{* 2}}{\epsilon_{\chi}^{* 2} \sqrt{\epsilon^{*} \epsilon_{\chi}^{*}}}\right. \\
& \left.-3 \frac{u^{2}}{\epsilon_{\phi}^{* 2}}\left(\frac{u}{\epsilon_{\phi}^{*}}-\frac{v}{\epsilon_{\chi}^{*}}\right) \eta_{\phi}^{*} \mathcal{A}-3 \frac{v^{2}}{\epsilon_{\chi}^{* 2}}\left(\frac{v}{\epsilon_{\chi}^{*}}-\frac{u}{\epsilon_{\phi}^{*}}\right) \eta_{\chi}^{*} \mathcal{A}+\left(\frac{u}{\epsilon_{\phi}^{*}}-\frac{v}{\epsilon_{\chi}^{*}}\right)^{3} \mathcal{B}^{2} .\right]
\end{aligned}
$$


We now rewrite the horizon crossing slow-roll parameters in terms of their kinematic counterparts such as $\eta_{s s}^{*}$. There are three kinematic $\eta$ parameters and four $\xi^{2}$ parameters which means that there is no unique way in which to do this. We shall choose to rewrite $\eta_{\phi}^{*}$ and $\eta_{\chi}^{*}$ in terms of $\eta_{s s}^{*}$ and $\eta_{\sigma s}^{*}$ and $\xi_{\phi}^{* 2}$ and $\xi_{\chi}^{* 2}$ in terms of $\xi_{s s s}^{*}{ }^{2}$ and $\xi_{\sigma s s}^{*}{ }^{2}$. This gives the relations

$$
\begin{array}{r}
\eta_{\phi}=\eta_{s s}-\sqrt{\frac{\epsilon_{\phi}}{\epsilon_{\chi}}} \eta_{\sigma s}, \\
\eta_{\chi}=\eta_{s s}+\sqrt{\frac{\epsilon_{\chi}}{\epsilon_{\phi}}} \eta_{\sigma s}, \\
\xi_{\phi}^{2}=\frac{\sqrt{\epsilon \epsilon_{\phi}}}{\epsilon_{\chi}} \xi_{\sigma s s}^{2}-\sqrt{\frac{\epsilon}{\epsilon_{\chi}}} \xi_{s s s}^{2}, \\
\xi_{\chi}^{2}=\frac{\sqrt{\epsilon \epsilon_{\chi}}}{\epsilon_{\phi}} \xi_{\sigma s s}^{2}+\sqrt{\frac{\epsilon}{\epsilon_{\phi}}} \xi_{s s s}^{2} .
\end{array}
$$

Substituting Eq. (A.15) into Eq. (A.14) and simplifying we find

$$
\begin{aligned}
& n_{s}-1=2\left(\eta_{s s}^{*}-\epsilon^{*}\right)-2\left(\frac{u^{2}}{\epsilon_{\phi}^{*}}+\frac{v^{2}}{\epsilon_{\chi}^{*}}\right)^{-1}\left[2+\frac{u-v}{\sqrt{\epsilon_{\phi}^{*} \epsilon_{\chi}^{*}}} \eta_{\sigma s}^{*}\right], \\
& f_{\mathrm{NL}}=\frac{5}{6}\left(\frac{u^{2}}{\epsilon_{\phi}^{*}}+\frac{v^{2}}{\epsilon_{\chi}^{*}}\right)^{-2}\left[2\left(\frac{u^{2}}{\epsilon_{\phi}^{*}}+\frac{v^{2}}{\epsilon_{\chi}^{*}}\right)-\left(\frac{u^{3}}{\epsilon_{\phi}^{* 2}}+\frac{v^{3}}{\epsilon_{\chi}^{* 2}}\right) \eta_{s s}^{*}\right. \\
& \left.+\left(\frac{u^{3}}{\epsilon_{\phi}^{*}}-\frac{v^{3}}{\epsilon_{\chi}^{*}}\right) \frac{\eta_{\sigma s}^{*}}{\sqrt{\epsilon_{\phi}^{*} \epsilon_{\chi}^{*}}}+2\left(\frac{u}{\epsilon_{\phi}^{*}}-\frac{v}{\epsilon_{\chi}^{*}}\right)^{2} \mathcal{A}\right], \\
& \tau_{\mathrm{NL}}=\left(\frac{u^{2}}{\epsilon_{\phi}^{*}}+\frac{v^{2}}{\epsilon_{\chi}^{*}}\right)^{-3}\left[\left(\frac{u^{4}}{\epsilon_{\phi}^{* 3}}+\frac{v^{4}}{\epsilon_{\chi}^{* 3}}\right) \eta_{s s}^{* 2}-2\left(\frac{u^{4}}{\epsilon_{\phi}^{* 2}}-\frac{v^{4}}{\epsilon_{\chi}^{* 2}}\right) \frac{\eta_{s s}^{*} \eta_{\sigma s}^{*}}{\sqrt{\epsilon_{\phi}^{*} \epsilon_{\chi}^{*}}}\right. \\
& +\left(\frac{u^{4}}{\epsilon_{\phi}^{*}}+\frac{v^{4}}{\epsilon_{\chi}^{*}}\right) \frac{\eta_{\sigma s}^{* 2}}{\epsilon_{\phi}^{*} \epsilon_{\chi}^{*}}-4\left(\frac{u^{3}}{\epsilon_{\phi}^{* 2}}+\frac{v^{3}}{\epsilon_{\chi}^{* 2}}\right) \eta_{s s}^{*}+4\left(\frac{u^{3}}{\epsilon_{\phi}^{*}}-\frac{v^{3}}{\epsilon_{\chi}^{*}}\right) \frac{\eta_{\sigma s}^{*}}{\sqrt{\epsilon_{\phi}^{*} \epsilon_{\chi}^{*}}} \\
& +4\left(\frac{u^{2}}{\epsilon_{\phi}^{*}}+\frac{v^{2}}{\epsilon_{\chi}^{*}}\right)-4\left(\frac{u}{\epsilon_{\phi}^{*}}-\frac{v}{\epsilon_{\chi}^{*}}\right)^{2}\left(\frac{u}{\epsilon_{\phi}^{*}}+\frac{v}{\epsilon_{\chi}^{*}}\right) \eta_{s s}^{*} \mathcal{A}+4\left(\frac{u}{\epsilon_{\phi}^{*}}-\frac{v}{\epsilon_{\chi}^{*}}\right)\left(\frac{u^{2}}{\epsilon_{\phi}^{*}}+\frac{v^{2}}{\epsilon_{\chi}^{*}}\right) \frac{\eta_{\sigma s}^{*} \mathcal{A}}{\sqrt{\epsilon_{\phi}^{*} \epsilon_{\chi}^{*}}} \\
& \left.+8\left(\frac{u}{\epsilon_{\phi}^{*}}-\frac{v}{\epsilon_{\chi}^{*}}\right)^{2} \mathcal{A}+4\left(\frac{u}{\epsilon_{\phi}^{*}}-\frac{v}{\epsilon_{\chi}^{*}}\right)^{2} \frac{\epsilon^{*} \mathcal{A}^{2}}{\epsilon_{\phi}^{*} \epsilon_{\chi}^{*}}\right], \\
& g_{\mathrm{NL}}=\frac{50}{54}\left(\frac{u^{2}}{\epsilon_{\phi}^{*}}+\frac{v^{2}}{\epsilon_{\chi}^{*}}\right)^{-3}\left[\left(\frac{u^{4}}{\epsilon_{\phi}^{* 3}}+\frac{v^{4}}{\epsilon_{\chi}^{* 3}}\right) \eta_{s s}^{* 2}-2\left(\frac{u^{4}}{\epsilon_{\phi}^{* 2}}-\frac{v^{4}}{\epsilon_{\chi}^{* 2}}\right) \frac{\eta_{s s}^{*} \eta_{\sigma s}^{*}}{\sqrt{\epsilon_{\phi}^{*} \epsilon_{\chi}^{*}}}\right. \\
& +\left(\frac{u^{4}}{\epsilon_{\phi}^{*}}+\frac{v^{4}}{\epsilon_{\chi}^{*}}\right) \frac{\eta_{\sigma s}^{*}}{\epsilon_{\phi}^{*} \epsilon_{\chi}^{*}}-\left(\frac{u^{3}}{\epsilon_{\phi}^{* 2}}+\frac{v^{3}}{\epsilon_{\chi}^{* 2}}\right) \eta_{s s}^{*}+\left(\frac{u^{3}}{\epsilon_{\phi}^{*}}-\frac{v^{3}}{\epsilon_{\chi}^{*}}\right) \frac{\eta_{\sigma s}^{*}}{\sqrt{\epsilon_{\phi}^{*} \epsilon_{\chi}^{*}}}
\end{aligned}
$$




$$
\begin{aligned}
& +\frac{1}{2}\left(\frac{u^{4}}{\epsilon_{\phi}^{* 2}}-\frac{v^{4}}{\epsilon_{\chi}^{* 2}}\right) \frac{\xi_{s s s}^{* 2}}{\sqrt{\epsilon_{\phi}^{*} \epsilon_{\chi}^{*}}}-\frac{1}{2}\left(\frac{u^{4}}{\epsilon_{\phi}^{*}}+\frac{v^{4}}{\epsilon_{\chi}^{*}}\right) \frac{\xi_{\sigma s s}^{*}}{\epsilon_{\phi}^{*} \epsilon_{\chi}^{*}} \\
& \left.-3\left(\frac{u}{\epsilon_{\phi}^{*}}-\frac{v}{\epsilon_{\chi}^{*}}\right)^{2}\left(\frac{u}{\epsilon_{\phi}^{*}}+\frac{v}{\epsilon_{\chi}^{*}}\right) \eta_{s s}^{*} \mathcal{A}+3\left(\frac{u}{\epsilon_{\phi}^{*}}-\frac{v}{\epsilon_{\chi}^{*}}\right)\left(\frac{u^{2}}{\epsilon_{\phi}^{*}}+\frac{v^{2}}{\epsilon_{\chi}^{*}}\right) \frac{\eta_{\sigma s}^{*} \mathcal{A}}{\sqrt{\epsilon_{\phi}^{*} \epsilon_{\chi}^{*}}}+\left(\frac{u}{\epsilon_{\phi}^{*}}-\frac{v}{\epsilon_{\chi}^{*}}\right)^{3} \mathcal{B}^{2}\right] .
\end{aligned}
$$

\section{A.2 Product-separable potentials}

For a potential with the two-field product-separable form $W=U(\phi) V(\chi)$, the first derivatives of $N$ are

$$
\begin{aligned}
M_{\mathrm{pl}} N_{, \phi}=\frac{u}{\sqrt{2 \epsilon_{\phi}^{*}}}, & u=\frac{\epsilon_{\phi}}{\epsilon}, \\
M_{\mathrm{pl}} N_{, \chi}=\frac{v}{\sqrt{2 \epsilon_{\chi}^{*}}}, & v=\frac{\epsilon_{\chi}}{\epsilon} .
\end{aligned}
$$

In the $\{\phi, \chi\}$ frame the potential is product-separable which means that any of the parameters $\eta_{i j}$ or $\xi_{i j k}^{2}$ with mixed derivatives can be written in terms of lower-order slow-roll parameters that do not have mixed derivatives. This again allows us to use the single-index notation $\eta_{\phi}$ and $\xi_{\phi}^{2}$ for the remaining terms. In the kinematic basis the three $\eta$ components are

$$
\begin{aligned}
\eta_{\sigma \sigma} & =\frac{\epsilon_{\phi} \eta_{\phi}+\epsilon_{\chi} \eta_{\chi}+4 \epsilon_{\phi} \epsilon_{\chi}}{\epsilon} \\
\eta_{\sigma s} & =\frac{\sqrt{\epsilon_{\phi} \epsilon_{\chi}}}{\epsilon}\left[\left(\eta_{\chi}-2 \epsilon_{\chi}\right)-\left(\eta_{\phi}-2 \epsilon_{\phi}\right)\right] \\
\eta_{s s} & =\frac{\epsilon_{\chi} \eta_{\phi}+\epsilon_{\phi} \eta_{\chi}-4 \epsilon_{\phi} \epsilon_{\chi}}{\epsilon}
\end{aligned}
$$

The components of the $\xi_{i j k}^{2}$ tensor are

$$
\begin{aligned}
\epsilon^{3 / 2} \xi_{\sigma \sigma \sigma}^{2} & =\epsilon_{\chi}^{3 / 2} \xi_{\chi}^{2}+\epsilon_{\phi}^{3 / 2} \xi_{\phi}^{2}+6 \epsilon_{\phi} \epsilon_{\chi} \sqrt{\epsilon}\left(\eta_{\phi}+\eta_{\chi}\right), \\
\epsilon^{3 / 2} \xi_{\sigma \sigma s}^{2} & =\epsilon_{\chi} \sqrt{\epsilon_{\phi}} \xi_{\chi}^{2}-\epsilon_{\phi} \sqrt{\epsilon_{\chi}} \xi_{\phi}^{2}+2 \sqrt{\epsilon \epsilon_{\phi} \epsilon_{\chi}}\left[\left(\epsilon_{\phi}-2 \epsilon_{\chi}\right) \eta_{\phi}-\left(\epsilon_{\chi}-2 \epsilon_{\phi}\right) \eta_{\chi}\right], \\
\epsilon^{3 / 2} \xi_{\sigma s s}^{2} & =\epsilon_{\phi} \sqrt{\epsilon_{\chi}} \xi_{\chi}^{2}+\epsilon_{\chi} \sqrt{\epsilon_{\phi}} \xi_{\phi}^{2}+2 \sqrt{\epsilon}\left[\left(\epsilon_{\phi}-2 \epsilon_{\chi}\right) \epsilon_{\phi} \eta_{\chi}+\left(\epsilon_{\chi}-2 \epsilon_{\phi}\right) \epsilon_{\chi} \eta_{\phi}\right], \\
\epsilon^{3 / 2} \xi_{s s s}^{2} & =\epsilon_{\phi}^{3 / 2} \xi_{\chi}^{2}-\epsilon_{\chi}^{3 / 2} \xi_{\phi}^{2}+6 \sqrt{\epsilon \epsilon_{\phi} \epsilon_{\chi}}\left(\epsilon_{\chi} \eta_{\phi}-\epsilon_{\phi} \eta_{\chi}\right) .
\end{aligned}
$$

The second derivatives of $N$ follow by differentiation of Eqs. (A.17), giving

$$
\begin{aligned}
& M_{\mathrm{pl}}^{2} N_{, \phi \phi}=u-\frac{u \eta_{\phi}^{*}}{2 \epsilon_{\phi}^{*}}+\frac{\mathcal{A}_{P}}{\epsilon_{\phi}^{*}}, \\
& M_{\mathrm{pl}}^{2} N_{, \phi \chi}=-\frac{\mathcal{A}_{P}}{\sqrt{\epsilon_{\phi}^{*} \epsilon_{\chi}^{*}}}, \\
& M_{\mathrm{pl}}^{2} N_{, \chi \chi}=v-\frac{v \eta_{\chi}^{*}}{2 \epsilon_{\chi}^{*}}+\frac{\mathcal{A}_{P}}{\epsilon_{\chi}^{*}},
\end{aligned}
$$

where we have substituted

$$
\mathcal{A}_{P} \equiv u v \eta_{s s}
$$


to put these equations into a form similar to that found for the sum-separable potential. Taking the next derivative we find

$$
\begin{aligned}
& M_{\mathrm{pl}}^{3} N_{, \phi \phi \phi}=\frac{1}{\epsilon_{\phi}^{*} \sqrt{2 \epsilon_{\phi}^{*}}}\left(-\frac{u}{2} \sqrt{\frac{\epsilon_{\phi}^{*}}{\epsilon^{*}}} \xi_{\phi}^{* 2}-u \epsilon_{\phi}^{*} \eta_{\phi}^{*}+u \eta_{\phi}^{* 2}-3\left(\eta_{\phi}^{*}-2 \epsilon_{\phi}^{*}\right) \mathcal{A}_{P}+\mathcal{B}_{P}^{2}\right), \\
& M_{\mathrm{pl}}^{3} N_{, \phi \phi \chi}=\frac{1}{\epsilon_{\phi}^{*} \sqrt{2 \epsilon_{\chi}^{*}}}\left(\left(\eta_{\phi}^{*}-2 \epsilon_{\phi}^{*}\right) \mathcal{A}_{P}-\mathcal{B}_{P}^{2}\right), \\
& M_{\mathrm{pl}}^{3} N_{, \phi \chi \chi}=\frac{1}{\epsilon_{\chi}^{*} \sqrt{2 \epsilon_{\phi}^{*}}}\left(\left(\eta_{\chi}^{*}-2 \epsilon_{\chi}^{*}\right) \mathcal{A}_{P}+\mathcal{B}_{P}^{2}\right), \\
& M_{\mathrm{pl}}^{3} N_{, \chi \chi \chi}=\frac{1}{\epsilon_{\chi}^{*} \sqrt{2 \epsilon_{\chi}^{*}}}\left(-\frac{v}{2} \sqrt{\frac{\epsilon_{\chi}^{*}}{\epsilon^{*}}} \xi_{\chi}^{* 2}-v \epsilon_{\chi}^{*} \eta_{\chi}^{*}+v \eta_{\chi}^{* 2}-3\left(\eta_{\chi}^{*}-2 \epsilon_{\chi}^{*}\right) \mathcal{A}_{P}-\mathcal{B}_{P}^{2}\right),
\end{aligned}
$$

where

$$
\begin{aligned}
M_{\mathrm{pl}} \sqrt{2 \epsilon_{\phi}^{*}} \frac{\partial \mathcal{A}_{P}}{\partial \phi_{*}} & \equiv-M_{\mathrm{pl}} \sqrt{2 \epsilon_{\chi}^{*}} \frac{\partial \mathcal{A}_{P}}{\partial \chi_{*}} \equiv \mathcal{B}_{P}^{2} \\
\mathcal{B}_{P}^{2} & \equiv-\sqrt{u v}^{3}\left[\xi_{s s s}^{2}+2 \frac{\epsilon_{\phi}-\epsilon_{\chi}}{\sqrt{\epsilon_{\phi} \epsilon_{\chi}}} \eta_{s s}^{2}-2 \eta_{\sigma s} \eta_{s s}\right] .
\end{aligned}
$$

As with the sum-separable case, $\mathcal{A}_{P}$ is symmetric under switching the fields whilst $\mathcal{B}_{P}^{2}$ is anti-symmetric. We take these results together to find

$$
\begin{aligned}
& n_{s}-1=-4\left(\frac{u^{2}}{\epsilon_{\phi}^{*}}+\frac{v^{2}}{\epsilon_{\chi}^{*}}\right)^{-1}\left[1-2 u v-\frac{u^{2} \eta_{\phi}^{*}}{2 \epsilon_{\phi}^{*}}-\frac{v^{2} \eta_{\chi}^{*}}{2 \epsilon_{\chi}^{*}}\right]-2 \epsilon^{*} \\
& f_{\mathrm{NL}}=\frac{5}{6}\left(\frac{u^{2}}{\epsilon_{\phi}^{*}}+\frac{v^{2}}{\epsilon_{\chi}^{*}}\right)^{-2}\left[2\left(\frac{u^{3}}{\epsilon_{\phi}^{*}}+\frac{v^{3}}{\epsilon_{\chi}^{*}}\right)-\frac{u^{3} \eta_{\phi}^{*}}{\epsilon_{\phi}^{* 2}}-\frac{v^{3} \eta_{\chi}^{*}}{\epsilon_{\chi}^{* 2}}+2\left(\frac{u}{\epsilon_{\phi}^{*}}-\frac{v}{\epsilon_{\chi}^{*}}\right)^{2} \mathcal{A}_{P}\right], \\
& \tau_{\mathrm{NL}}=4\left(\frac{u^{2}}{\epsilon_{\phi}^{*}}+\frac{v^{2}}{\epsilon_{\chi}^{*}}\right)^{-3}\left[-\frac{u^{4} \eta_{\phi}^{*}}{\epsilon_{\phi}^{* 2}}-\frac{v^{4} \eta_{\chi}^{*}}{\epsilon_{\chi}^{* 2}}+\frac{u^{4} \eta_{\phi}^{* 2}}{4 \epsilon_{\phi}^{* 3}}+\frac{v^{4} \eta_{\chi}^{* 2}}{4 \epsilon_{\chi}^{* 3}}+\frac{u^{4}}{\epsilon_{\phi}^{*}}+\frac{v^{4}}{\epsilon_{\chi}^{*}}\right. \\
& -\frac{u^{2}}{\epsilon_{\phi}^{* 2}}\left(\frac{u}{\epsilon_{\phi}^{*}}-\frac{v}{\epsilon_{\chi}^{*}}\right) \eta_{\phi}^{*} \mathcal{A}_{P}-\frac{v^{2}}{\epsilon_{\chi}^{* 2}}\left(\frac{v}{\epsilon_{\chi}^{*}}-\frac{u}{\epsilon_{\phi}^{*}}\right) \eta_{\chi}^{*} \mathcal{A}_{P} \\
& \left.+2\left(\frac{u^{2}}{\epsilon_{\phi}^{*}}-\frac{v^{2}}{\epsilon_{\chi}^{*}}\right)\left(\frac{u}{\epsilon_{\phi}^{*}}-\frac{v}{\epsilon_{\chi}^{*}}\right) \mathcal{A}_{P}+\left(\frac{u}{\epsilon_{\phi}^{*}}-\frac{v}{\epsilon_{\chi}^{*}}\right)^{2}\left(\frac{1}{\epsilon_{\phi}^{*}}+\frac{1}{\epsilon_{\chi}^{*}}\right) \mathcal{A}_{P}^{2}\right], \\
& g_{\mathrm{NL}}=\frac{50}{54}\left(\frac{u^{2}}{\epsilon_{\phi}^{*}}+\frac{v^{2}}{\epsilon_{\chi}^{*}}\right)^{-3}\left[-\frac{u^{4} \eta_{\phi}^{*}}{\epsilon_{\phi}^{* 2}}-\frac{v^{4} \eta_{\chi}^{*}}{\epsilon_{\chi}^{* 2}}+\frac{u^{4} \eta_{\phi}^{* 2}}{\epsilon_{\phi}^{* 3}}+\frac{v^{4} \eta_{\chi}^{* 2}}{\epsilon_{\chi}^{* 3}}-\frac{1}{2} \frac{u^{4} \xi_{\phi}^{* 2}}{\epsilon_{\phi}^{* 2} \sqrt{\epsilon^{*} \epsilon_{\phi}^{*}}}-\frac{1}{2} \frac{v^{4} \xi_{\chi}^{* 2}}{\epsilon_{\chi}^{* 2} \sqrt{\epsilon^{*} \epsilon_{\chi}^{*}}}\right. \\
& -3 \frac{u^{2}}{\epsilon_{\phi}^{* 2}}\left(\frac{u}{\epsilon_{\phi}^{*}}-\frac{v}{\epsilon_{\chi}^{*}}\right) \eta_{\phi}^{*} \mathcal{A}_{P}-3 \frac{v^{2}}{\epsilon_{\chi}^{* 2}}\left(\frac{v}{\epsilon_{\chi}^{*}}-\frac{u}{\epsilon_{\phi}^{*}}\right) \eta_{\chi}^{*} \mathcal{A}_{P} \\
& \left.+6\left(\frac{u^{2}}{\epsilon_{\phi}^{*}}-\frac{v^{2}}{\epsilon_{\chi}^{*}}\right)\left(\frac{u}{\epsilon_{\phi}^{*}}-\frac{v}{\epsilon_{\chi}^{*}}\right) \mathcal{A}_{P}+\left(\frac{u}{\epsilon_{\phi}^{*}}-\frac{v}{\epsilon_{\chi}^{*}}\right)^{3} \mathcal{B}_{P}^{2} .\right] .
\end{aligned}
$$


One can rewrite the horizon crossing slow-roll parameters in terms of their kinematic counterparts as

$$
\begin{aligned}
& \eta_{\phi}=\eta_{s s}+2 \epsilon_{\phi}-\sqrt{\frac{\epsilon_{\phi}}{\epsilon_{\chi}}} \eta_{\sigma s}, \\
& \eta_{\chi}=\eta_{s s}+2 \epsilon_{\chi}+\sqrt{\frac{\epsilon_{\chi}}{\epsilon_{\phi}}} \eta_{\sigma s}, \\
& \xi_{\phi}^{2}=\frac{\sqrt{\epsilon \epsilon_{\phi}}}{\epsilon_{\chi}} \xi_{\sigma s s}^{2}-\sqrt{\frac{\epsilon}{\epsilon_{\chi}}} \xi_{s s s}^{2}-2 \frac{\sqrt{\epsilon \epsilon_{\phi}}}{\epsilon_{\chi}}\left(\epsilon_{\phi} \eta_{\chi}-2 \epsilon_{\chi} \eta_{\phi}\right), \\
& \xi_{\chi}^{2}=\frac{\sqrt{\epsilon \epsilon_{\chi}}}{\epsilon_{\phi}} \xi_{\sigma s s}^{2}+\sqrt{\frac{\epsilon}{\epsilon_{\phi}}} \xi_{s s s}^{2}-2 \frac{\sqrt{\epsilon \epsilon_{\chi}}}{\epsilon_{\phi}}\left(\epsilon_{\chi} \eta_{\phi}-2 \epsilon_{\phi} \eta_{\chi}\right) .
\end{aligned}
$$

Substituting Eq. (A.29) into Eq. (A.28) and simplifying we find

$$
\begin{aligned}
& n_{s}-1=2\left(\eta_{s s}^{*}-\epsilon^{*}\right)-2\left(\frac{u^{2}}{\epsilon_{\phi}^{*}}+\frac{v^{2}}{\epsilon_{\chi}^{*}}\right)^{-1} \frac{u-v}{\sqrt{\epsilon_{\phi}^{*} \epsilon_{\chi}^{*}}} \eta_{\sigma s}^{*}, \\
& f_{\mathrm{NL}}=\frac{5}{6}\left(\frac{u^{2}}{\epsilon_{\phi}^{*}}+\frac{v^{2}}{\epsilon_{\chi}^{*}}\right)^{-2}\left[-\left(\frac{u^{3}}{\epsilon_{\phi}^{* 2}}+\frac{v^{3}}{\epsilon_{\chi}^{* 2}}\right) \eta_{s s}^{*}+\left(\frac{u^{3}}{\epsilon_{\phi}^{*}}-\frac{v^{3}}{\epsilon_{\chi}^{*}}\right) \frac{\eta_{\sigma s}^{*}}{\sqrt{\epsilon_{\phi}^{*} \epsilon_{\chi}^{*}}}+2\left(\frac{u}{\epsilon_{\phi}^{*}}-\frac{v}{\epsilon_{\chi}^{*}}\right)^{2} \mathcal{A}_{P}\right] \text {, } \\
& \tau_{\mathrm{NL}}=\left(\frac{u^{2}}{\epsilon_{\phi}^{*}}+\frac{v^{2}}{\epsilon_{\chi}^{*}}\right)^{-3}\left[\left(\frac{u^{4}}{\epsilon_{\phi}^{* 3}}+\frac{v^{4}}{\epsilon_{\chi}^{* 3}}\right) \eta_{s s}^{* 2}-2\left(\frac{u^{4}}{\epsilon_{\phi}^{* 2}}-\frac{v^{4}}{\epsilon_{\chi}^{* 2}}\right) \frac{\eta_{s s}^{*} \eta_{\sigma s}^{*}}{\sqrt{\epsilon_{\phi}^{*} \epsilon_{\chi}^{*}}}\right. \\
& +\left(\frac{u^{4}}{\epsilon_{\phi}^{*}}+\frac{v^{4}}{\epsilon_{\chi}^{*}}\right) \frac{\eta_{\sigma s}^{*}{ }^{2}}{\epsilon_{\phi}^{*} \epsilon_{\chi}^{*}}-4\left(\frac{u}{\epsilon_{\phi}^{*}}-\frac{v}{\epsilon_{\chi}^{*}}\right)^{2}\left(\frac{u}{\epsilon_{\phi}^{*}}+\frac{v}{\epsilon_{\chi}^{*}}\right) \eta_{s s}^{*} \mathcal{A}_{P} \\
& \left.+4\left(\frac{u}{\epsilon_{\phi}^{*}}-\frac{v}{\epsilon_{\chi}^{*}}\right)\left(\frac{u^{2}}{\epsilon_{\phi}^{*}}+\frac{v^{2}}{\epsilon_{\chi}^{*}}\right) \frac{\eta_{\sigma s}^{*} \mathcal{A}_{P}}{\sqrt{\epsilon_{\phi}^{*} \epsilon_{\chi}^{*}}}+4\left(\frac{u}{\epsilon_{\phi}^{*}}-\frac{v}{\epsilon_{\chi}^{*}}\right)^{2} \frac{\epsilon^{*} \mathcal{A}_{P}^{2}}{\epsilon_{\phi}^{*} \epsilon_{\chi}^{*}}\right], \\
& g_{\mathrm{NL}}=\frac{50}{54}\left(\frac{u^{2}}{\epsilon_{\phi}^{*}}+\frac{v^{2}}{\epsilon_{\chi}^{*}}\right)^{-3}\left[\left(\frac{u^{4}}{\epsilon_{\phi}^{* 3}}+\frac{v^{4}}{\epsilon_{\chi}^{* 3}}\right) \eta_{s s}^{* 2}-2\left(\frac{u^{4}}{\epsilon_{\phi}^{* 2}}-\frac{v^{4}}{\epsilon_{\chi}^{* 2}}\right) \frac{\eta_{s s}^{*} \eta_{\sigma s}^{*}}{\sqrt{\epsilon_{\phi}^{*} \epsilon_{\chi}^{*}}}\right. \\
& +\left(\frac{u^{4}}{\epsilon_{\phi}^{*}}+\frac{v^{4}}{\epsilon_{\chi}^{*}}\right) \frac{\eta_{\sigma s}^{* 2}}{\epsilon_{\phi}^{*} \epsilon_{\chi}^{*}}+\left(\frac{u^{4}}{\epsilon_{\phi}^{*}}+\frac{v^{4}}{\epsilon_{\chi}^{*}}\right) \frac{\epsilon^{*} \eta_{s s}^{*}}{\epsilon_{\phi}^{*} \epsilon_{\chi}^{*}} \\
& +\frac{1}{2}\left(\frac{u^{4}}{\epsilon_{\phi}^{* 2}}-\frac{v^{4}}{\epsilon_{\chi}^{* 2}}\right) \frac{\xi_{s s s}^{*}{ }^{2}}{\sqrt{\epsilon_{\phi}^{*} \epsilon_{\chi}^{*}}}-\frac{1}{2}\left(\frac{u^{4}}{\epsilon_{\phi}^{*}}+\frac{v^{4}}{\epsilon_{\chi}^{*}}\right) \frac{\xi_{\sigma s s}^{*}}{\epsilon_{\phi}^{*} \epsilon_{\chi}^{*}} \\
& \left.-3\left(\frac{u}{\epsilon_{\phi}^{*}}-\frac{v}{\epsilon_{\chi}^{*}}\right)^{2}\left(\frac{u}{\epsilon_{\phi}^{*}}+\frac{v}{\epsilon_{\chi}^{*}}\right) \eta_{s s}^{*} \mathcal{A}_{P}+3\left(\frac{u}{\epsilon_{\phi}^{*}}-\frac{v}{\epsilon_{\chi}^{*}}\right)\left(\frac{u^{2}}{\epsilon_{\phi}^{*}}+\frac{v^{2}}{\epsilon_{\chi}^{*}}\right) \frac{\eta_{\sigma s}^{*} \mathcal{A}_{P}}{\sqrt{\epsilon_{\phi}^{*} \epsilon_{\chi}^{*}}}+\left(\frac{u}{\epsilon_{\phi}^{*}}-\frac{v}{\epsilon_{\chi}^{*}}\right)^{3} \mathcal{B}_{P}^{2}\right] .
\end{aligned}
$$

\section{B Simplification of Trispectrum expressions}

The function $\left|\tau_{6}\right|$, as defined in Eq. (3.13), is bounded well within our limit of ten and so this term may be immediately neglected. Furthermore, we can use Eq. (3.6) to manipulate 
various other terms. In the sum-separable case we find

$$
\begin{aligned}
\tau_{2} \eta_{s s}^{*} \eta_{\sigma s}^{*}= & {\left[\sin 2 \theta^{*} \tau_{2}\right] \eta_{s s}^{*} \eta_{\sigma s}^{*}+\left[\left(1-\sin 2 \theta^{*}\right) \tau_{2} \frac{1}{2} \tan 2 \theta^{*}\right] \eta_{s s}^{*}\left(\eta_{s s}^{*}-\eta_{\sigma \sigma}^{*}\right), } \\
\tau_{3} \eta_{\sigma s}^{* 2}= & {\left[\sin ^{2} 2 \theta^{*} \tau_{3}\right] \eta_{\sigma s}^{* 2}+\left[\left(1-\sin ^{2} 2 \theta^{*}\right) \tau_{3} \frac{1}{4} \tan ^{2} 2 \theta^{*}\right]\left(\eta_{s s}^{*}-\eta_{\sigma \sigma}^{*}\right)^{2} } \\
\tau_{5} \epsilon^{*} \eta_{\sigma s}^{*}= & {\left[\sin 2 \theta^{*} \tau_{5}\right] \epsilon^{*} \eta_{\sigma s}^{*}+\left[\left(1-\sin 2 \theta^{*}\right) \tau_{5} \frac{1}{2} \tan 2 \theta^{*}\right] \epsilon^{*}\left(\eta_{s s}^{*}-\eta_{\sigma \sigma}^{*}\right), } \\
-\tau_{8} \Omega \eta_{\sigma s}^{*}\left(\eta_{s s}-\epsilon\right)=- & {\left[\sin ^{2} 2 \theta^{*} \tau_{8}\right] \Omega \eta_{\sigma s}^{*}\left(\eta_{s s}-\epsilon\right) } \\
& -\left[\left(1-\sin 2 \theta^{*}\right) \tau_{8} \frac{1}{2} \tan 2 \theta^{*}\right] \Omega\left(\eta_{s s}^{*}-\eta_{\sigma \sigma}^{*}\right)\left(\eta_{s s}-\epsilon\right),
\end{aligned}
$$

and we find that all of the functions in square brackets never have a magnitude greater than ten and so these terms represent variations in the trispectrum that are significantly smaller than observables will ever probe. One can easily check that these results follow analogously for product-separable potentials and so these terms may be neglected for both types of separable potential.

Analogously to Eq. (3.6) there exist formulae relating the various $\xi_{i j k}^{2}$ components. For sum-separable potentials we have

$$
\begin{aligned}
& \xi_{\sigma s s}^{2}=\frac{1}{2} \tan 2 \theta\left(\xi_{s s s}^{2}-\xi_{\sigma \sigma s}^{2}\right), \\
& \xi_{\sigma \sigma s}^{2}=\frac{1}{2} \tan 2 \theta\left(\xi_{\sigma s s}^{2}-\xi_{\sigma \sigma \sigma}^{2}\right),
\end{aligned}
$$

whereas for product-separable potentials these relations are of the form

$$
\begin{aligned}
& \xi_{\sigma s s}^{2}=\frac{1}{2} \tan 2 \theta\left(\xi_{s s s}^{2}-\xi_{\sigma \sigma s}^{2}\right)+2 \epsilon \eta_{s s}+2 \tan 2 \theta \epsilon \eta_{\sigma s}, \\
& \xi_{\sigma \sigma s}^{2}=\frac{1}{2} \tan 2 \theta\left(\xi_{\sigma s s}^{2}-\xi_{\sigma \sigma \sigma}^{2}\right)-2 \epsilon \eta_{\sigma s}+2 \tan 2 \theta \epsilon\left(\eta_{s s}+\epsilon\right) .
\end{aligned}
$$

Using Eqs. (B.2) and (B.3) we can then simplify the full expression for $g_{\mathrm{NL}}$ in Eq. (3.10) as

$$
\begin{aligned}
\frac{1}{4} \tau_{2} \xi_{s s s}^{* 2}-\frac{1}{2} \tau_{3} \xi_{\sigma s s}^{* 2}= & \frac{1}{4} \tau_{2} \xi_{s s s}^{* 2}-\sin ^{2} 2 \theta^{*} \frac{1}{2} \tau_{3} \xi_{\sigma s s}^{* 2}-\left(1-\sin ^{2} 2 \theta^{*}\right) \frac{1}{2} \tau_{3} \\
& \times\left(\frac{1}{2} \tan 2 \theta^{*} \xi_{s s s}^{*} 2-\frac{1}{4} \tan ^{2} 2 \theta^{*}\left(\xi_{\sigma s s}^{*}{ }^{2}-\xi_{\sigma \sigma \sigma}^{*}{ }^{2}\right)\right) \\
= & -g_{4} \xi_{s s s}^{* 2}-\left[\frac{1}{2} \tau_{3} \sin ^{2} 2 \theta^{*}\right]\left(\frac{3}{4} \xi_{\sigma s s}^{*}{ }^{2}+\frac{1}{4} \xi_{\sigma \sigma \sigma}^{*}{ }^{2}\right) \\
\simeq & -g_{4} \xi_{s s s}^{*}{ }^{2}
\end{aligned}
$$

where we have defined $g_{4}=\frac{1}{4}\left(\tau_{3} \sin 2 \theta^{*} \cos 2 \theta^{*}-\tau_{2}\right)$ in the expressions above. The term in square brackets in the last line can never be large and so is neglected.

The product-separable case follows similarly, yielding the same answer, however the calculation is unsurprisingly more involved. Manipulating three of the terms in Eq. (3.12) for $g_{\mathrm{NL}}$ by using Eq. (B.4) we find

$$
\begin{aligned}
\frac{1}{4} \tau_{2} \xi_{s s s}^{* 2}+\tau_{3} \epsilon^{*} \eta_{s s}^{*}-\frac{1}{2} \tau_{3} \xi_{\sigma s s}^{* 2}=- & g_{4} \xi_{s s s}^{* 2}-\left[\frac{1}{2} \tau_{3} \sin ^{2} 2 \theta^{*}\right]\left(\xi_{\sigma s s}^{*}{ }^{2}-2 \epsilon^{*} \eta_{s s}^{*}\right) \\
& +\frac{1}{4} \tau_{3} \sin 2 \theta^{*} \cos 2 \theta^{*}\left(\xi_{\sigma \sigma s}^{*}{ }^{2}-4 \epsilon^{*} \eta_{\sigma s}^{*}\right) .
\end{aligned}
$$


We now expand the last term of Eq. (B.7) by substituting for $\xi_{\sigma \sigma s}^{*}{ }^{2}$ using Eq. (B.5). We also use Eq. (3.6) to rewrite $\eta_{\sigma s}^{*}$ as $\sin 2 \theta^{*} \eta_{\sigma s}^{*}+\left(1-\sin 2 \theta^{*}\right) \frac{1}{2} \tan 2 \theta^{*}\left(\eta_{s s}-\eta_{\sigma \sigma}+2 \epsilon\right)$ and so we ultimately find

$$
\begin{aligned}
& \frac{1}{4} \tau_{2} \xi_{s s s}^{* 2}+\tau_{3} \epsilon^{*} \eta_{s s}^{*}- \frac{1}{2} \tau_{3} \xi_{\sigma s s}^{* 2}=-g_{4} \xi_{s s s}^{* 2}-\left[\frac{1}{2} \tau_{3} \sin ^{2} 2 \theta^{*}\right]\left(\frac{3}{4} \xi_{\sigma s s}^{*}{ }^{2}+\frac{1}{4} \xi_{\sigma \sigma \sigma}^{*}{ }^{2}-3 \epsilon^{*} \eta_{s s}^{*}\right. \\
&\left.-\epsilon^{* 2}+3 \epsilon^{*} \eta_{\sigma s}^{*} \cos 2 \theta^{*}+\frac{3}{2}\left(1-\sin 2 \theta^{*}\right)\left(\eta_{s s}^{*}-\eta_{\sigma \sigma}^{*}+2 \epsilon^{*}\right)\right)
\end{aligned}
$$

The term in square brackets is always negligible and this multiplies terms no larger than $\mathcal{O}\left(\epsilon^{* 2}\right)$ and so may be ignored, leaving the same simple result that we found for sum-separable potentials.

After these various terms have been neglected from Eqs. (3.9) to (3.12), we can simplify the remaining terms by rewriting them by means of the trigonometric relations

$$
\begin{aligned}
\tau_{1} & =\tau+2 f+1, \\
\tau_{4} & =2 f_{1}(1+f), \\
\tau_{7} & =4(\tau+f), \\
2 g_{2} & =\tau-f .
\end{aligned}
$$

\section{References}

[1] J. M. Maldacena, Non-Gaussian features of primordial fluctuations in single field inflationary models, JHEP 05 (2003) 013, [astro-ph/0210603].

[2] D. Seery and J. E. Lidsey, Primordial non-gaussianities from multiple-field inflation, JCAP 0509 (2005) 011, [astro-ph/0506056].

[3] D. Seery, J. E. Lidsey, and M. S. Sloth, The inflationary trispectrum, JCAP 0701 (2007) 027, [astro-ph/0610210].

[4] D. Seery, M. S. Sloth, and F. Vernizzi, Inflationary trispectrum from graviton exchange, JCAP 0903 (2009) 018, [arXiv:0811.3934].

[5] J. M. Bardeen, P. J. Steinhardt, and M. S. Turner, Spontaneous Creation of Almost Scale-Free Density Perturbations in an Inflationary Universe, Phys.Rev. D28 (1983) 679.

[6] D. Lyth, Large Scale Energy Density Perturbations and Inflation, Phys.Rev. D31 (1985) 1792-1798.

[7] G. I. Rigopoulos and E. P. S. Shellard, The Separate Universe Approach and the Evolution of Nonlinear Superhorizon Cosmological Perturbations, Phys. Rev. D68 (2003) 123518, [astro-ph/0306620].

[8] D. H. Lyth, K. A. Malik, and M. Sasaki, A general proof of the conservation of the curvature perturbation, JCAP 0505 (2005) 004, [astro-ph/0411220].

[9] S. Weinberg, Must cosmological perturbations remain non-adiabatic after multi-field inflation?, Phys. Rev. D70 (2004) 083522, [astro-ph/0405397].

[10] D. Langlois and F. Vernizzi, Evolution of non-linear cosmological perturbations, Phys. Rev. Lett. 95 (2005) 091303, [astro-ph/0503416].

[11] D. Langlois and F. Vernizzi, Conserved non-linear quantities in cosmology, Phys. Rev. D72 (2005) 103501, [astro-ph/0509078].

[12] D. Salopek, J. Bond, and J. M. Bardeen, Designing Density Fluctuation Spectra in Inflation, Phys.Rev. D40 (1989) 1753. 
[13] D. Polarski and A. A. Starobinsky, Isocurvature perturbations in multiple inflationary models, Phys. Rev. D50 (1994) 6123-6129, [astro-ph/9404061].

[14] M. Sasaki and E. D. Stewart, A General analytic formula for the spectral index of the density perturbations produced during inflation, Prog. Theor. Phys. 95 (1996) 71-78, [astro-ph/9507001].

[15] J. Garcia-Bellido and D. Wands, Metric perturbations in two-field inflation, Phys. Rev. D53 (1996) 5437-5445, [astro-ph/9511029].

[16] D. S. Salopek, Characteristics of cosmic time, Phys. Rev. D52 (1995) 5563-5575, [astro-ph/9506146].

[17] D. Langlois, Correlated adiabatic and isocurvature perturbations from double inflation, Phys. Rev. D59 (1999) 123512, [astro-ph/9906080].

[18] C. Gordon, D. Wands, B. A. Bassett, and R. Maartens, Adiabatic and entropy perturbations from inflation, Phys. Rev. D63 (2001) 023506, [astro-ph/0009131].

[19] D. Langlois and F. Vernizzi, A geometrical approach to nonlinear perturbations in relativistic cosmology, Class. Quant. Grav. 27 (2010) 124007, [arXiv:1003.3270].

[20] D. J. Mulryne, D. Seery, and D. Wesley, Moment transport equations for non-Gaussianity, JCAP 1001 (2010) 024, [arXiv:0909.2256].

[21] D. J. Mulryne, D. Seery, and D. Wesley, Moment transport equations for the primordial curvature perturbation, JCAP 1104 (2011) 030, [arXiv:1008.3159].

[22] M. Dias and D. Seery, Transport equations for the inflationary spectral index, Phys.Rev. D85 (2012) 043519, [arXiv:1111.6544].

[23] Planck Collaboration, Planck: The scientific programme, astro-ph/0604069.

[24] G. I. Rigopoulos, E. P. S. Shellard, and B. J. W. van Tent, Quantitative bispectra from multifield inflation, Phys. Rev. D76 (2007) 083512, [astro-ph/0511041].

[25] F. Vernizzi and D. Wands, Non-Gaussianities in two-field inflation, JCAP 0605 (2006) 019, [astro-ph/0603799].

[26] L. Alabidi, Non-gaussianity for a two component hybrid model of inflation, JCAP 0610 (2006) 015, [astro-ph/0604611].

[27] C. T. Byrnes, K.-Y. Choi, and L. M. H. Hall, Conditions for large non-Gaussianity in two-field slow- roll inflation, JCAP 0810 (2008) 008, [arXiv:0807.1101].

[28] D. H. Lyth and Y. Rodriguez, The inflationary prediction for primordial non- gaussianity, Phys. Rev. Lett. 95 (2005) 121302, [astro-ph/0504045].

[29] D. Seery and J. E. Lidsey, Non-gaussianity from the inflationary trispectrum, JCAP 0701 (2007) 008, [astro-ph/0611034].

[30] C. T. Byrnes, M. Sasaki, and D. Wands, The primordial trispectrum from inflation, Phys. Rev. D74 (2006) 123519, [astro-ph/0611075].

[31] J. Meyers and N. Sivanandam, Adiabaticity and the Fate of Non-Gaussianities: The Trispectrum and Beyond, Phys. Rev. D84 (2011) 063522, [arXiv:1104.5238].

[32] WMAP Collaboration, E. Komatsu et. al., Seven-Year Wilkinson Microwave Anisotropy Probe (WMAP) Observations: Cosmological Interpretation, Astrophys. J. Suppl. 192 (2011) 18, [arXiv: 1001.4538].

[33] J. Smidt et. al., CMB Constraints on Primordial non-Gaussianity from the Bispectrum $\left(f_{N L}\right)$ and Trispectrum $\left(g_{N L}\right.$ and $\left.\tau_{N L}\right)$ and a New Consistency Test of Single-Field Inflation, Phys. Rev. D81 (2010) 123007, [arXiv:1004.1409]. 
[34] J. Smidt et. al., A Measurement of Cubic-Order Primordial Non-Gaussianity ( $g_{N L}$ and $\tau_{N L}$ ) With WMAP 5-Year Data, arXiv:1001.5026.

[35] J. R. Fergusson, D. M. Regan, and E. P. S. Shellard, Optimal Trispectrum Estimators and WMAP Constraints, arXiv:1012.6039.

[36] E. Komatsu, Hunting for Primordial Non-Gaussianity in the Cosmic Microwave Background, Class. Quant. Grav. 27 (2010) 124010, [arXiv:1003.6097].

[37] T. Suyama and M. Yamaguchi, Non-Gaussianity in the modulated reheating scenario, Phys. Rev. D77 (2008) 023505, [arXiv:0709.2545].

[38] J. Elliston, D. J. Mulryne, D. Seery, and R. Tavakol, Evolution of $f N L$ to the adiabatic limit, JCAP 1111 (2011) 005, [arXiv:1106.2153].

[39] J. Elliston, D. Mulryne, D. Seery, and R. Tavakol, Evolution of non-Gaussianity in multi-scalar field models, Int. J. Mod. Phys. A26 (2011) 3821-3832, [arXiv:1107.2270].

[40] C. M. Peterson and M. Tegmark, Testing Two-Field Inflation, Phys. Rev. D83 (2011) 023522, [arXiv: 1005.4056].

[41] C. M. Peterson and M. Tegmark, Non-Gaussianity in Two-Field Inflation, Phys. Rev. D84 (2011) 023520, [arXiv:1011.6675].

[42] C. M. Peterson and M. Tegmark, Testing Multi-Field Inflation: A Geometric Approach, arXiv: 1111.0927.

[43] K. A. Malik and D. Wands, Cosmological perturbations, Phys. Rept. 475 (2009) 1-51, [arXiv:0809.4944].

[44] D. Wands, K. A. Malik, D. H. Lyth, and A. R. Liddle, A new approach to the evolution of cosmological perturbations on large scales, Phys. Rev. D62 (2000) 043527, [astro-ph/0003278].

[45] A. A. Starobinsky, Multicomponent de Sitter (Inflationary) Stages and the Generation of Perturbations, JETP Lett. 42 (1985) 152-155.

[46] T. Battefeld and R. Easther, Non-gaussianities in multi-field inflation, JCAP 0703 (2007) 020, [astro-ph/0610296].

[47] K.-Y. Choi, L. M. H. Hall, and C. van de Bruck, Spectral running and non-Gaussianity from slow-roll inflation in generalised two-field models, JCAP 0702 (2007) 029, [astro-ph/0701247].

[48] T. Wang, Note on Non-Gaussianities in Two-field Inflation, Phys. Rev. D82 (2010) 123515, [arXiv: 1008.3198].

[49] C. T. Byrnes, K.-Y. Choi, and L. M. H. Hall, Large non-Gaussianity from two-component hybrid inflation, JCAP 0902 (2009) 017, [arXiv:0812.0807].

[50] J. Meyers and N. Sivanandam, Non-Gaussianities in Multifield Inflation: Superhorizon Evolution, Adiabaticity, and the Fate of fnl, Phys. Rev. D83 (2011) 103517, [arXiv: 1011.4934].

[51] S. A. Kim, A. R. Liddle, and D. Seery, Non-gaussianity in axion Nflation models, Phys. Rev. Lett. 105 (2010) 181302, [arXiv: 1005.4410].

[52] D. Mulryne, S. Orani, and A. Rajantie, Non-Gaussianity from the hybrid potential, Phys. Rev. D84 (2011) 123527, [arXiv:1107.4739].

[53] S. A. Kim, A. R. Liddle, and D. Seery, Non-gaussianity in axion N-flation models: detailed predictions and mass spectra, Phys. Rev. D85 (2012) 023532, [arXiv:1108.2944].

[54] D. Seery, D. J. Mulryne, J. Frazer, and R. H. Ribeiro, Inflationary perturbation theory is geometrical optics in phase space, arXiv:1203.2635.

[55] I. Huston and A. J. Christopherson, Calculating Non-adiabatic Pressure Perturbations during 
Multi-field Inflation, Phys. Rev. D85 (2012) 063507, [arXiv:1111.6919].

[56] S. A. Kim and A. R. Liddle, Nflation: Non-gaussianity in the horizon-crossing approximation, Phys. Rev. D74 (2006) 063522, [astro-ph/0608186].

[57] D. H. Lyth, Generating the curvature perturbation at the end of inflation, JCAP 0511 (2005) 006, [astro-ph/0510443].

[58] N. S. Sugiyama, Consistency Relation for multifield inflation scenario with all loop contributions, arXiv:1201.4048.

[59] A. D. Linde, Axions in inflationary cosmology, Phys.Lett. B259 (1991) 38-47.

[60] L. Alabidi and D. Lyth, Curvature perturbation from symmetry breaking the end of inflation, JCAP 0608 (2006) 006, [astro-ph/0604569].

[61] M. P. Salem, On the generation of density perturbations at the end of inflation, Phys. Rev. D72 (2005) 123516, [astro-ph/0511146].

[62] M. Sasaki, Multi-brid inflation and non-Gaussianity, Prog. Theor. Phys. 120 (2008) 159-174, [arXiv:0805.0974].

[63] A. Naruko and M. Sasaki, Large non-Gaussianity from multi-brid inflation, Prog. Theor. Phys. 121 (2009) 193-210, [arXiv:0807.0180].

[64] Q.-G. Huang, A geometric description of the non-Gaussianity generated at the end of multi-field inflation, JCAP 0906 (2009) 035, [arXiv:0904.2649].

[65] A. A. Abolhasani and H. Firouzjahi, No Large Scale Curvature Perturbations during Waterfall of Hybrid Inflation, Phys. Rev. D83 (2011) 063513, [arXiv:1005.2934].

[66] T. Suyama, T. Takahashi, M. Yamaguchi, and S. Yokoyama, On Classification of Models of Large Local-Type Non- Gaussianity, JCAP 1012 (2010) 030, [arXiv:1009.1979].

[67] D. Mulryne, D. Seery, and D. Wesley, Non-Gaussianity constrains hybrid inflation, arXiv:0911.3550.

[68] K.-Y. Choi, S. A. Kim, and B. Kyae, Primordial curvature perturbation during and at the end of multi-field inflation, arXiv:1202.0089.

[69] D. H. Lyth, The hybrid inflation waterfall and the primordial curvature perturbation, arXiv: 1201.4312.

[70] R. Jeannerot, Inflation in supersymmetric unified theories, Phys. Rev. D56 (1997) 6205-6216, [hep-ph/9706391].

[71] R. Jeannerot, J. Rocher, and M. Sakellariadou, How generic is cosmic string formation in SUSY GUTs, Phys. Rev. D68 (2003) 103514, [hep-ph/0308134].

[72] N. Bevis, M. Hindmarsh, M. Kunz, and J. Urrestilla, Fitting CMB data with cosmic strings and inflation, Phys. Rev. Lett. 100 (2008) 021301, [astro-ph/0702223].

[73] N. Bevis, M. Hindmarsh, M. Kunz, and J. Urrestilla, CMB power spectra from cosmic strings: predictions for the Planck satellite and beyond, Phys. Rev. D82 (2010) 065004, [arXiv: 1005.2663].

[74] F. Bernardeau and J.-P. Uzan, Inflationary models inducing non-gaussian metric fluctuations, Phys. Rev. D67 (2003) 121301, [astro-ph/0209330].

[75] F. Bernardeau, L. Kofman, and J.-P. Uzan, Modulated fluctuations from hybrid inflation, Phys. Rev. D70 (2004) 083004, [astro-ph/0403315]. 